\title{
A Sustainable Approach of Damping Treatment for Aluminium Honeycomb Sandwich Structures
}

\author{
P Monninger and A N Thite
}

School of Engineering, Computing and Mathematics, Oxford Brookes University, Oxford, OX33 1HX, UK

\section{Abstract}

The damping plays a vital role in structural dynamic and acoustic performance of aluminium honeycomb sandwich structures. The viscoelastic damping treatment of skins is most common. An alternative, the use of sustainable cork inserts to improve the damping of cores and the whole assembly is investigated in this study. Structures with different filling degrees are analysed as well as the optimum location for inserts is determined. The structural dynamic as well as the vibro-acoustic performance is estimated numerically. Average squared displacement amplitude reduction efficiency $\overline{E_{\overline{d^{2}}}}$ is defined as the target parameter for structural dynamic performance, whereas average transmission loss effectiveness $\overline{E_{T L}}$ for vibro-acoustic performance. The structural dynamic models are validated by experimental vibration analysis, whereas the vibro-acoustic models are validated against published data. Different ways of bonding the inserts to the host structure are analysed in order to maximise damping. The highest improvement is obtained with a filling degree of $64 \%$ honeycomb voids and $9.76 \%$ increase in mass, for which an average squared displacement amplitude reduction of $35.25 \%$ and an average increase in transmission loss of $1.5 \mathrm{~dB}$ is achieved. The transmission loss increase in relation to the added mass is much higher than that achieved by doubling of mass in the mass law region. The introduction of cork inserts spreads the energy in local modes to a larger space, effectively decreasing the resonance amplitudes. Interestingly, damping does not increase with the number of inserts in a monotonic way and the improvement depends on the spatial distribution of inserts. 
Key words: Honeycomb sandwich, Cork, Core insert, Damping, Structural dynamic,

Structural-acoustic, Transmission loss, Aluminium core

Correspondence: athite@,brookes.ac.uk, Ph: +44 1865484320. 


\section{Introduction}

The use of light-weight structures is becoming increasingly important in the automotive field. One such light weight technology is the application of sandwich materials such as honeycomb sandwich structures. These are widely used in light-weight engineering and hence are not only increasingly used in the automotive sector [1] but have been prominent for long in shipbuilding [2], aerospace [3], and transport packaging [4]. Although, honeycomb structures provide good structural properties, their damping behaviour is mostly poor compared to structures with a higher volumetric density, leading to higher structural and acoustic response. This study concerns the use of cork as an insert for aluminium core sandwiches and ensuing improvement in the structural dynamic and structural-acoustic performance.

The vibration of structures can be controlled by additional damping. The damping can be increased by using viscoelastic material [5] or foam [6], for example. There have been many investigations (for example [7-10]) concerning approaches to improve structural dynamic performance of honeycomb structures, in particular. Several studies have been carried out on core filled honeycomb sandwich structures. The mechanisms of dissipation such as viscoelastic and friction are used. The honeycomb cores containing particles dissipate vibrational energy because of friction and other complex mechanisms [7]. This area of research continues of interest; further studies to improve or predict the performance of the cores with particles have been carried out [11 - 13]. Viscoelastic inserts in honeycomb cores have been used for the same purpose with good degree of effectiveness. The cores have been filled with rubber [14-15] and foam [6-8] to increase the damping.

Cork has been used (for example the study by Santo Silva et al. [16]) to improve dynamic performance in sandwich structures; the strength advantage in to the use of cork is minimal but the improvement in damping can be very large. There has been no investigation in the use of cork as an insert in aluminium core, very stiff sandwich structures. The use of cork 
has a huge advantage as being lightweight and can be sustainably manufactured. In this study the damping performance of an aluminium honeycomb sandwich structure will be optimised using cork inserts.

Arunkumar et al. [17] determined the sound radiation and transmission loss characteristics of a honeycomb sandwich panel made with composite fibres and aluminium sandwich panels. Reducing the transmitted sound energy through a honeycomb sandwich structure by attaching gas layers to the structure was studied by Naify et al. [18]. Zhou and Crocker [19] looked into the sound transmission loss of honeycomb sandwich panels filled with foam. Overall, only a few studies can be found optimising acoustic performance of honeycomb structures using internal damping devices.

Choosing the optimum location of the inserts throughout the structure has a huge impact on the overall damping behaviour [20]. Different approaches were used to determine the optimum location of damping inserts in order to increase damping accounting for increased mass. Fotsing et al. [21] showed that the optimum location for damping inserts was close to vibration nodes for which shear strains reach their maximum. Boucher et al. [20] concluded that a partial occupation of the voids could be more efficient in terms of added weight. A parametric optimisation and the adaptive indicator-based evolutionary algorithm was employed by Aumjaud et al. [22] in selecting damping insertion locations, focussing on the first two mode shapes.

On the other hand, the cork is widely used as an environmental friendly core material for lightweight sandwich structures. Studies show that it is not only an ideal core material in terms of mechanical strength and impact resistance [23], it also improves damping performance significantly. Flexural behaviour of a composite sandwich structure with a core made out of agglomerated cork has been analysed by Kumar et al. [24]. Sargianis et al. [25] created 
sandwich composites without affecting mechanical performance or weight in a negative way but increasing damping by as much as $250 \%$.

In this study, three different types of structures with varying configuration of cork inserts will be analysed. The structural arrangements are : (a) an empty host structure (EHS) without any inserts, (b) a simply damped structure (SDS) for which inserts are distributed equally over the structure and fill 30\% voids, and (c) structures for which inserts are placed in specific voids to achieve maximum efficiency and hence to identify the optimally damped structure (ODS). The damping efficiency of all structures is analysed by defining target parameters for both, structural and acoustic response, considering the added mass. The structural dynamic as well as the vibro-acoustic performance of the structures is estimated using numerical simulations. First, the optimum surface bonding of inserts within the host structure is determined, followed by the validation of the basic numerical models. Based on this, the location of the inserts is optimised, leading to possible variants of insert arrangements. The structural dynamic and vibro-acoustic performance of all structural variants is then analysed. Having only one insert placed in the centre void is identified as the most efficient structure. Due to the very small increase in mass and relatively larger reduction in vibration displacement as well as a significant increase in transmission loss (TL) very high efficiency values are achieved. However, most improved performance is for a filling degree of $64 \%$. The introduction of cork inserts spreads the energy in local modes to a larger region, effectively decreasing the resonance amplitudes of the aluminium core. Additionally, it is shown that damping does not increase with the number of inserts in a monotonic way and improvement is highly dependent on location of the inserts. Finally, the findings are compared against different damping approaches in the literature. 


\section{Structural Dynamic and Structural-Acoustic Modelling}

In this study the structural dynamic performance of honeycomb sandwich with cork inserts is estimated by a numerical approach. For finite element modelling, a commercial software, ANSYS is used. The finite element models use quadrilateral elements and the element density is sufficient to capture the mode shapes accurately. The results of numerical analysis are then validated by comparison with experimental frequency response functions. The structural-acoustic performance is estimated by quantifying the transmission loss using the numerical approach and validated against baseline published results from the literature.

The honeycomb sandwich structures show complex structural dynamic behaviour. The low frequency behaviour is dominated by global modes that are dependent on the equivalent bending stiffness of construction. For example, Figure 1a shows one such mode where global behaviour can be seen. The core shear and rotation contribute at higher frequencies, resulting in localised modes. The localised modes are standing waves that are dependent on the core depth. Figure $1 \mathrm{~b}$ shows one such mode - in these modes there is a very efficient transfer of vibrational energy across the honeycomb sandwich. The use of constrained or unconstrained layer damping helps reduce the global modal response. The localised core behaviour is not significantly affected by this type of damping. The higher frequencies can be influenced by damping of core structure using inserts. The overall effect would be the reduction of velocity response of the panels, eventually reducing the structure-borne sound.

\section{Figure 1}

The damping in honeycomb sandwiches also influences the airborne transmission of acoustic waves; the resonance region in the transmission loss curve just after the stiffness controlled region and as well as the coincidence region are affected. The coincidence region shows broader frequency range because of the orthotropic behaviour of the core. This 
orthotropic behaviour emphasises the need for increased damping at higher frequencies over a wider frequency band.

The details of the honeycomb sandwich panel preparation, numerical analysis, experimental measurements and performance indicators are given in this section.

\subsection{Baseline Assembly}

The height of the honeycomb core is $10 \mathrm{~mm}$ and consists out of 77 cells. The distance between parallel sides of each cell is $19.1 \mathrm{~mm}$ and the foil has a thickness of $50 \mu \mathrm{m}$. The overall sandwich structure is symmetrical and has a length of $171.95 \mathrm{~mm}$, a width of $154.3 \mathrm{~mm}$, and a height of $12.4 \mathrm{~mm}$ (see Figure $2 \mathrm{a}$ ). The adhesive used for bonding the structures is Araldite ${ }^{\circledR}$ Standard. For simply damping (SDS), in addition 23 inserts are placed equally distributed within the host structure (see Figure 2b). Initial damping values for aluminium and cork are obtained from literature based on the damping model introduced in section 2.2.3. The structural parameters can be found in Table 1.

Figure 2.

Table 1.

\subsection{Structural Dynamic Performance}

\subsubsection{Optimum Surface Bonding}

Based on the initial material parameters (see Table 1), the optimum bonding condition of inserts within the voids is determined by frequency response functions generated numerically. Different combinations are assessed as shown in Figure 2, where shaded areas 
indicate bonded faces. Three combinations are studied: (a) connecting the inserts to the outer panels without bonding to the core (Figure 3a), (b) connecting the inserts only to the core (Figure 3b), and (c) connecting the inserts to the core and one of the panels (see Figure 3c). Apart from imparting additional damping the inserts may influence the effective mass and stiffness of the sandwich panel. Connecting the inserts directly to the outer panels is expected to result in direct transfer of energy through the cross section of the insert - it is of interest to find effect on the stiffness and mass compared with the additional damping, in particular, on the global modes. In the second case, connecting the inserts only to the core allows shearing action in the inserts - the damping improvement therefore can be significant in this case. As well, this may influence the effective stiffness and mass in a complex way. The additional mass now acts through the stiffness related to shearing action as well as the core stiffness. The core stiffness may also increase as the flexural standing waves, as seen in Figure $1 \mathrm{~b}$, are resisted by the presence of inserts. The third option is a combination of the first two cases; it is an option which can be easily manufactured.

\section{Figure 3.}

\subsubsection{Experimental Vibration Analysis}

For the EHS as well as the SDS five samples were manufactured. The testing arrangement can be seen in Figure 4. There are five designated locations for measurement: four on the front face (shown as black dots in the figure) and one on the back face. The accelerance frequency response function was measured.

\section{Figure 4.}

Instrumented impact hammer was used to excite the structure and a piezoelectric accelerometer was used to measure the acceleration response. The measurements were sampled at 20480 per second. A frequency range of $10240 \mathrm{~Hz}$ with a resolution $1.25 \mathrm{~Hz}$ is used. To reduce the 
measurement noise 10 averages were used to obtain averaged frequency response function. Exponential window is used on the acceleration response.

To determine the damping ratio of the modes the response from the location on the back panel was used for measurement so that the drive point frequency response function can be generated. The lower modes are expected to be well separated and hence half-power bandwidth method was used to estimate the modal damping ratios.

\subsubsection{Structural Dynamic Performance Parameter}

The forced vibration analysis was performed using a unit harmonic force. The frequency resolution of $1 \mathrm{~Hz}$ was used throughout in the numerical analysis. The frequency response function, accelerance, was determined numerically by inversion of a dynamic stiffness matrix at every frequency. Rayleigh's model of damping with two parameters is the most common approach used in the forced vibration analysis. However, in many practical cases the performance of this model deviates based on the way the parameters are estimated. To reduce the potential errors, extra parameters can be used to append the Rayleigh's approach. In commercial software some hybrid models are used. One such model available in ANSYS, which is an extension of Rayleigh's damping model, has an additional element of constant damping. The associated damping matrix is given by

$$
\mathbf{C}=\sum_{k=1}^{n} \alpha_{k} \mathbf{M}_{k}+\sum_{k=1}^{n}\left(\beta_{k}+\frac{1}{\omega} g_{k}\right) \mathbf{K}_{k}
$$

where $n$ is the number of materials in the model, $\alpha$ and $\beta$ are Rayleigh model's constants multiplying the mass matrix $(\mathbf{M})$ and the stiffness matrix $(\mathbf{K})$ respectively, $\omega$ is the excitation frequency and $g$ is the constant, frequency independent structural damping ratio. The parameters of the model are related to a modal damping ratio as given below. 


$$
\xi_{i}=\frac{\alpha}{2 \omega_{i}}+\frac{\beta \omega_{i}}{2}+\frac{g}{2}
$$

where $\omega_{i}$ is the natural frequency of the $i^{\text {th }}$ mode. The parameters of this damping model were adjusted based on trial and error approach.

The squared displacement response is chosen as the performance indicator for assessing vibration performance. To be consistent with further acoustic analysis, the data is represented in octave bands. The target parameter is average squared displacement reduction efficiency for the $\mathrm{i}^{\text {th }} 1 / 3$ octave band, $E_{\overline{d^{2}}, i}$ which is given by

$$
E_{\overline{d^{2}, i}}=\frac{\Delta \overline{d^{2}}}{\Delta m_{a}}
$$

where $\Delta \bar{d}^{2}{ }_{i}$ is the average of squared displacement reduction of the measurement panel for the $\mathrm{i}^{\text {th }} 1 / 3$ octave band relative to the EHS and $\Delta m_{a}$ is the additional mass of the inserts as a proportion of the host structure's mass. The arithmetic mean over all $1 / 3$ octave bands of the analysed frequency range is then used to determine the overall structural dynamic performance

$$
\begin{aligned}
& \overline{E_{\overline{d^{2}}}}=\frac{1}{n} \sum_{I} E_{\overline{d^{2}}, i} \\
& =\frac{1}{n \times \Delta m_{a}} \sum_{I} \Delta{\overline{d^{2}}}_{i}
\end{aligned}
$$

\subsubsection{Damping Optimisation - Cluster Allocation}

To identify the optimum locations for inserts, the squared displacement response, $d^{2}$ is used as the performance parameter. Based on simply supported EHS the maximum displacement of the mid-node of each void for all modes is computed over a discrete frequency range from 0 to $10 \mathrm{kHz}$. 


$$
d_{i}^{2}=\max _{f} d_{i, j}^{2}
$$

where $d_{i}{ }^{2}$ is the maximum squared displacement for cell $i$ for all modes $j$ over the frequency range $f$. In order to cluster the cells according to their responsiveness, the span between largest and smallest $d_{i}{ }^{2}$ is divided into five equal sized levels which form five clusters. All cells are assigned to one of the five clusters according to their $d_{i}{ }^{2}$. The five clusters are named as CL1, CL2, CL3, CL4 and CL5; further details of which will be discussed later in the paper. The cells in these clusters are then filled with inserts starting with the most responsive cluster CL5. The optimum damping scheme is found when the target parameters of structural dynamic as well as vibro-acoustic performance reach their maximum.

\subsection{Vibro-Acoustic Performance Estimation}

The acoustic Transmission loss, TL of a barrier can be expressed as

$$
\mathrm{TL}=10 \times \log _{10}\left(\frac{I_{i}}{I_{t}}\right)
$$

where $I_{i}$ is the incident sound intensity and $I_{t}$ is the transmitted sound intensity. In most practical cases, to measure sound transmission loss adjacent reverberation room and anechoic room are used. The structure for which transmission loss is required is placed in a window between these rooms, acting as a barrier. The sound source is used on the reverberation room side to generate incident wavefield on the structure. In a reverberant room the sound intensity in terms of sound pressure is given by

$$
I_{\mathrm{i}}=\frac{\left\langle p^{2}\right\rangle}{4 \rho_{0} c_{0}}
$$


where $\left\langle p^{2}\right\rangle$ is the space averaged square of sound pressure, $\rho_{0}$ is the density of the air in the reverberation room and $c_{0}$ is the velocity of the sound in the air. The sound transmitted into the anechoic room can be quantified by the measurement of sound power. The sound power is then used to find the transmitted sound intensity as

$$
I_{\mathrm{t}}=\frac{W_{\mathrm{t}}}{S}
$$

where $W_{\mathrm{t}}$ is the sound power as measured in the anechoic room and $S$ is the surface area of the honeycomb sandwich exposed to anechoic room.

A similar arrangement of reverberation room and anechoic room combination can be created in commercially available finite element software to estimate the transmission loss. Incident sound is created by a diffuse sound field up to an angle of $90^{\circ}$ to the incident surface of the structure (see Figure 5a). The sound field is defined by a mesh-free reference hemisphere with a recommended radius of 50 times the length of the structure [26]. The energy of the diffuse sound field is uniformly distributed in all directions on the hemisphere's surface which is equally divided into 20 elementary surfaces. Within the receiving domain, which has anechoic condition, the transmitted sound intensity can be measured and the transmission loss curve can be computed.

\section{Figure 5.}

In order to take the fluid-structure interaction into account a coupled acoustic analysis was carried out. A multibody part and a 'Fluid-Structure Interaction' (FSI) boundary condition was applied to all surfaces of the structure interacting with the acoustic domain. Only the first layer of acoustic fluid elements connected to the structure are needed to be solved by a coupled 
algorithm - this is a computationally intensive task. For all other acoustic elements an uncoupled algorithm was chosen. In ANSYS, FLUID221 element is used where FSI condition exists. These elements discretize 3-D wave equation taking into consideration the coupling acoustic pressure and structural motion at the interface. The uncoupled zone that extends from the FLUID221 outer surface until the envelope of the room was meshed by FLUID220 elements, which discretise 3-D wave equation without structural interaction. Figure 5b shows meshed vibro-acoustic model using these elements.

Acoustic analysis was carried out under simply supported conditions which was achieved by applying zero displacement supports in all directions at two of the lower edges of the excited panels. The other two lower edges were constrained with a zero displacement in the direction orthogonal to the panel surface. This offers the advantage of having pressure variations at the shared edges of the structure with the acoustic domain. The receiving domain was designed as anechoic chamber which means the sound propagates into infinity and no reflection takes place. This was done by applying a radiation boundary to the outer surface of the acoustic domain. To improve computational efficiency, a symmetric solving algorithm was chosen for the acoustic domain for which only the upper triangle half of the matrices need to be stored during the solving process and hence avoids an in increase in CPU time by about 50 $\%$ [26]. The acoustic energy dissipation was neglected, and the fluid was assumed to be nonflowing as well as compressible.

The size of the domain is based on the recommendation that the outer surface region should be separated from the nearest object by at least 0.2 times the acoustic wavelength [26]. Hence, the radius of the hemisphere $r$ is given by

$$
r=\sqrt{L_{S}^{2}+W_{S}^{2}}+0.2 \times \lambda
$$


where $L_{S}$ is the length of the structure, $W_{S}$ is the width and $\lambda$ is the acoustic wavelength. The radius is dynamic since it is dependent on the respective acoustic wavelength. In order to cover all bending waves within the structures, the element size of the structure depends on the maximum excitation frequency $f_{\max }$. Hence, the minimum wavelength $\lambda_{\min }$ for the structure is

$$
\lambda_{\min }=\frac{c_{0}}{f_{\max }}
$$

where $c_{0}$ is the speed of sound in the fluid. To represent the bending waves accurately, it is recommended to have six elements per wavelength [26]. A dynamic mesh for the fluid was generated which means, that the element length is dependent on the respective frequency in order to generate the most economic mesh.

For acoustic barriers, the doubling of mass increases TL by $6 \mathrm{~dB}$ in the mass law region. The mass normalised improvement, therefore, is $0.06 \mathrm{~dB}$ per percentage increase in mass. The effectiveness in increasing transmission loss can be assessed by comparing mass normalised TL for the cork insert sandwich with that of TL increase in barriers when the mass is doubled. Therefore, the structural-acoustic performance indicator, the transmission loss effectiveness $E_{T L, i}$, is defined as

$$
E_{T L, i}=\frac{\Delta \mathrm{TL}_{i}}{0.06 \times \Delta m_{a}}
$$

where $\Delta T L_{i}$ is the transmission loss increase over EHS in $\mathrm{i}^{\text {th }} 1 / 3$ octave band. The arithmetic mean, over all $1 / 3$ octave bands, of the analysed frequency range is used to determine the overall vibro-acoustic performance.

$$
\overline{E_{T L}}=\frac{1}{n} \sum_{I} E_{T L, i}
$$




\subsection{Model Validation}

The validation of the numerical vibration models was carried out in two stages. First the material properties of aluminium were validated followed by the validation of the material properties of the inserts. The validation of material properties of the aluminium core and panels is based on the EHS. Since damping of the adhesive is allocated to the outer panels, the constant structural damping ratio $g_{\text {Panel }}$ of the panels was altered until the damping ratios match the ones obtained by physical experiments. To counter the change in stiffness and density due to adhesive the Young's Modulus of the aluminium core material $E_{\text {Core }}$ and the density of the panels $\rho_{\text {Panel }}$ were altered until the frequency of the modes match the experimental ones. It must be noted that only global modes were considered since local modes cannot be measured in experiments. The same principle was employed to validate the damping properties of the inserts. Validation was based on the SDS, where the inserts are uniformly distributed. All three initial damping parameters of cork were altered. The structural parameters of cork were validated by altering Young's Modulus and density of cork, $E_{\text {Cork }}$ and $\rho_{\text {Cork }}$.

The numerical acoustic model was validated by data from the literature. Since the work is unique, there is no published data about the structures studied here. However, as the models of the structures are validated already, it is reasonable to replace them with simple plates for which published data is readily available.

\section{Results and Discussion}

\subsection{Optimum Surface Bonding}

The initial damping values for the aluminium panels and the honeycomb core were computed based on data from Colakoglu and Jerina [27]. They determined damping ratios for discrete frequencies of aluminium panels. Using trial and error approach, based on the damping 
model of equation (2), the damping parameters were obtained (see Table 2). The damping ratios of cork NL $10 \circledR$ were taken from results of a study conducted by Silva et al. [28].

Table 2.

Structural dynamic performance was estimated for all combinations with point force excitation; Figure 6a shows numerically estimated accelerance amplitude as a function of frequency. The amplitudes at first few resonances show varied performance of insert bonding. Interestingly, the SDS for which inserts are connected to both panels shows less damping than the EHS for a few modes below about $3000 \mathrm{~Hz}$. This can be explained by the fact that vibrational energy is transmitted directly from the excited panel to the other panel. Even though the inserts provide damping, the overall transferred energy increases. Where inserts are connected to the core, improvement in damping can be achieved. This is due to the fact that the vibrating core causes shear stresses within the inserts. These stresses are damped and hence the overall vibration response. The change in stiffness and mass due to inserts shows a complex variation. The combination where the inserts are connected to panels does not significantly change the first natural frequency (global mode) compared with the structure without any inserts. However, for the case where the inserts are only connected to the core significant increase in the first natural frequency is seen. This may be due to relatively smaller increase in the effective mass due to the indirect connection of inserts to the panel (as discussed in Section 2.2.1) compared to significant increase in the effective stiffness of core. It must be noted that the overall damping increases with frequency since damping of cork increases with frequency $\left(\beta_{\text {Cork }}>0\right)$.

The best results can be achieved in connecting the inserts to the core only. However, with the existing laboratory setup, this was not feasible in terms of manufacturing. Therefore, in the study to find the optimum bonding combination the inserts were connected to the core and to one panel. This variant can be manufactured by bonding the core to one panel first. After 
the adhesive is hardened, the inserts can be bonded inside the core and then the second panel attached to the structure.

\section{Figure 6.}

\subsection{Model Validation}

For the EHS the first eight modes were used in the evaluation. The average error of damping ratio of the overall structure for the first eight modes is defined as the target value. Table 3 lists the modal damping ratios of structure without inserts and the one with the inserts. The assembled honeycomb structure shows significantly larger damping compared with aluminium material. The increase may be due to the joints formed by bonding. The lower frequency modes for the structures without and with inserts shows almost similar modal damping ratios; the performance, however, improves for the structure with inserts at higher modes as seen from Figure 6a. Since the half power bandwidth method is used in estimating the damping ratios, the accuracy depends on the frequency resolution. In general, it depends on two aspects: a) whether the resonance frequency measured is a true value, if not the peak value can be smaller and b) how small is the ratio of frequency resolution to the natural frequency compared with the damping ratio. There is some uncertainty, particularly for lower modes, as to the true peak at the resonance as it can only be measured to the accuracy of frequency resolution. For the other aspect, in this study the first mode is $\sim 1300 \mathrm{~Hz}$ and the resolution is $1.25 \mathrm{~Hz}$, therefore resulting in the ratio of 0.00096 , which is much smaller than the listed damping ratio values of the first modes in Table 3 . In any case, higher frequency behaviour is less sensitive to frequency resolution. The improved damping due to cork insert is further confirmed by the measured accelerances as shown in Figure $6 \mathrm{~b}$. The plot shows a broadband representation to indicate overall effectiveness at higher frequencies. 
The deviations in damping ratios from experiment to the numerical ones can be accounted by updating the model. Here again at lower frequencies the damping ratios are affected by the frequency resolution both in numerical and experimental studies. Since the resolution is similar in fineness for both the cases $(1 \mathrm{~Hz}$ for numerical and $1.25 \mathrm{~Hz}$ for experimental), error involved due to this can be considered minimal. While updating, for example the case without the cork inserts, as discussed in section 2.1, the increased damping can be accounted by additional material damping of the outer panels. Increasing $g_{\text {Panel }}$ from 0 to 0.045 the average error for 8 modes can be reduced to $2 \%$, which is seen to be sufficiently accurate. Figure 7 shows the comparison of experimental and numerical results for the structure without any inserts. In the narrow band representation (Figure 7a) small deviations are still seen, but for the vibro-acoustic study it is average over the frequency bands that is more critical. Figure $7 \mathrm{~b}$ shows $1 / 3^{\text {rd }}$ octave representation where the accelerance curves are seen almost identical.

\section{Figure 7.}

Damping ratios at lower frequencies for the SDS are similar for experimental and numerical studies, therefore, no further alteration of $g_{\text {Cork }}$ is necessary. The results of numerical model of SDS in Figure 8 are based on the updated panel damping. The frequency response functions are of similar shape and the numerical simulations are fairly accurate, hence no changes for $E_{\text {Core }}$ and $\rho_{\text {Panel }}$ as well as in $E_{\text {Cork }}$ and $\rho_{\text {Cork }}$ are required.

\section{Figure 8.}

Villot et al. [29] provide experimental transmission loss data of a simply supported aluminium plate with incident diffuse sound. In this study, the same plate was used for the numerical transmission loss model, for the purpose of validation. To better match experimental data, the angle of the incident diffuse sound field was limited to an angle between $70^{\circ}$ and $85^{\circ}$ ([30 - 32]); a maximum angle of $78^{\circ}$ was taken. Following the study of Villot et al. [29] 1/3 
octave bands with centre frequencies from $100 \mathrm{~Hz}$ to $5,000 \mathrm{~Hz}$ were analysed. Ten frequencies per $1 / 3$ octave band were used. The results can be seen in Figure 9.

\section{Figure 9.}

At lower frequencies, especially for the dip in TL for the first mode, the model is producing slightly different values. At higher frequencies the model matches the experimental results very well. The overall error is well below $1 \mathrm{~dB}$.

\subsection{Damping Optimisation}

On basis of the EHS, the displacement response of all cells over all global modes up to 10,000 Hz was analysed. This was done under simply supported conditions. The consideration of contribution from first 24 modes gives a stable cluster allocation, which is shown in Figure 10 where cluster five contains the most responsive cells. Interestingly, the allocation is only symmetrical for the clusters five and four (clusters are shown as CL1, CL2 _.. CL5). Since modes occur geometrically symmetrical, the cluster allocation was expected of similar nature. However, looking into the field of optimisation studies for damping of honeycomb structures, both symmetric as well as asymmetric solutions are found depending on the respective critical parameters. Asymmetric results are produced by Zheng et al. and Hou et al. [33 -34] for simply supported honeycomb structures. Both were using vibrational energy as a performance parameter which is related to the displacement response used in this study.

Figure 10.

\subsubsection{Structural Dynamic Performance}

The structural dynamic performance was assessed by exciting all structures at 16 points on one panel and measuring the response at 16 points on the opposite panel. Since for lower 
frequencies no huge effect of the cork inserts is expected, the analysis range starts from 1,000 $\mathrm{Hz}$ and reaches up to $10,000 \mathrm{~Hz}$. This is done to ensure a good representation of the response of the overall structure. The relative change in average squared displacement and added mass compared to the EHS as well as the computed target parameter $E_{\overline{\mathrm{d}^{2}}, \mathrm{i}}$ over all $1 / 3$ octave bands can be seen in Figure 11. The most effective structure is CL2 for which the displacement reduction parameter. $\Delta \overline{d^{2}}$, is the highest with $35.25 \%$. The filling degree of $64 \%$ translates to an increase in mass of $9.76 \%$. On the contrary, with only one insert placed in the centre void, CL5 shows the smallest value of $\Delta \overline{d^{2}}$ with $8.46 \%$. Comparing the SDS with the cluster structures, larger displacement reduction, $\Delta \overline{d^{2}}$, can be obtained for some structures having fewer cork inserts. This underlines the importance of the location selection on the structural dynamic performance. Interestingly, for the cluster structures, $\Delta \overline{d^{2}}$ does not increase with the number of inserts in a monotonic way. Although inserts do damp vibrations of the host structure, they do contribute towards effective stiffness and mass. Therefore, especially for CL1 compared to CL2, vibration response damped by additional inserts is exceeded by the vibration response caused by change in mass and stiffness. In terms of efficiency, , although reduction in displacement is not the highest, CL5 clearly performs best and hence is identified as ODS (optimally damped structure) for structural dynamic performance. The high value of 42.44 of $\overline{E_{\overline{d^{2}}}}$ is achieved due the small increase in mass, of only $0.20 \%$, but considerable increase in $\Delta \overline{d^{2}}$. Overall, the values for $\Delta \overline{d^{2}}$ and hence $\overline{E_{\overline{d^{2}}}}$ of all structures are positive, meaning that inserts do improve the structural dynamic performance.

\section{Figure 11.}

In order to get deeper insight into how improvement of structural dynamic performance is achieved, the average accelerance amplitude of the EHS and the most effective structure (CL2) in reducing displacement are plotted (see Figure 12a). Figure b shows the change in acceleration amplitude of CL5, SDS, and CL2 compared to the EHS. Due to the added weight 
of the inserts the modes do shift towards lower frequencies, causing increased amplitudes for certain frequencies. Nevertheless, acceleration amplitudes mostly decreased, indicating an improved performance. Improvement can be found especially for frequencies between 2 to 3 $\mathrm{kHz}$ and 5 to $7 \mathrm{kHz}$. In these regions local modes of either the resonating core or resonating inserts occur. This aligns with the findings of Mead ([35-36]) who investigated the harmonic wave propagation within one- and two-dimensional infinite periodic systems showing that modes exist in groups, so-called bands. On the other hand, there are frequency regions where bandgaps exist. Whereas, for the EHS many independent local modes of the core are generated, they are overlapped due to the presence of inserts in CL2. Additionally, the resonance amplitudes decrease. Both facts contribute to an increased performance of structures with cork inserts.

Figure 12.

\subsubsection{Vibro-Acoustic Performance}

As previously discussed in section 3.3.1, since the first resonance of the structures occurs at $\sim 1.3 \mathrm{kHz}$, a frequency range from 1 to $10 \mathrm{kHz}$ was analysed. Twenty points per $1 / 3$ octave band were computed in order to get a good resolution. Figure 13 shows the relative change in the TL and added mass compared to the EHS as well as the average transmission loss efficiency, $\overline{E_{T L}}$. Similar results to the ones for structural dynamic performance are obtained. Again, CL2 is the most effective structure with an average increase in TL of $1.5 \mathrm{~dB}$, whereas CL5 performs most efficiently with an increase of $0.6 \mathrm{~dB}$ in TL and a efficiency value of 51.23

for $\overline{E_{T L}}$. Hence, CL5 is designated as ODS also in terms of vibro-acoustic performance. The CL2 performance is 2.57 times as good as that by doubling of mass in a single leaf barrier.

Figure 13. 
Looking into the behaviour of the EHS and CL2 over the frequency range, the effect of modal behaviour is very clear (see Figure 14a). Due to the increased damping a small improvement in TL is seen over most of the frequency range. At very low frequencies, as seen in the structural dynamic response, occasionally the TL is smaller than that for EHS. However, the improvement is pronounced and consistent, especially after $3 \mathrm{kHz}$. In Figure $14 \mathrm{~b}$ the relative change in TL of CL5, SDS and CL2 compared to the EHS is shown. For all three structures a similar behaviour can be seen, however, the performance is more pronounced for the structure with CL2 insert configuration.

\section{Figure 14.}

\subsection{Comparison with Literature}

The results obtained are compared against findings of three different studies (see Figure 15). These studies, however, concern only the first few modes. Therefore, the current findings may seem smaller due to being averaged over a wider frequency range where a number of modes contribute to the response. Damping by cork inserts achieves better efficiency for nearly all filling degrees compared to particles used by Wang and Yang [12]. Their host structure is assembled out of a paper-resin honeycomb core covered by carbon fibre sheets. Fine spherical solder balls were chosen as damping particles; values for $\Delta \overline{d^{2}}$ up to $92 \%$ could be achieved, which is far better than for cork in this study. However, their poor efficiency is mainly caused by the significant increase in mass. Hence, only their structure with a smallest filling degree shows a better value for $\overline{E_{\overline{d^{2}}}}$ compared to CL1.

Nia and Sadeghi [10] investigated the damping efficiency of five different foam filled aluminium honeycomb structures in an axial loading-experimental study; they analysed the 
absorbed energy which is proportional to the squared displacement and hence comparable. Even though they add more mass to the overall structures, the values achieved for displacement reduction efficiency, $\overline{E_{\overline{d^{2}}}}$, are located in a similar range to CL3, CL2, CL1 and SDS. In turn, this leads to the conclusion that far more energy is absorbed by foam than by cork.

Using foam, again, Woody and Smith [8] achieved the highest efficiency value. However, CL5 provides comparable performance. Again, the host structure is made of aluminium honeycomb material. Since the same level of added mass as of cork is reached, the better energy absorption of foam compared to cork is proven. The material used is, however, non-sustainable and not economical.

Although cork seems to have poorer energy absorption capability compared to particles and foam, two major differences in the studies must be noted. First, in the studies compared a narrower frequency range and only the first few modes have been used. Since damping affects the dominant resonance amplitudes the strongest, far better results can be presented. Wang and Yang [12] did only study the first two global modes, whereas Woody and Smith [8] analysed the first five resonance frequencies. Second, cork inserts achieve good damping results by damping the local modes of the core. Hence, a fair comparison needs to look into the energy absorption from local modes over a larger frequency range. Since this data is not provided in the compared work, a very clear reasoning about which damping material performs is difficult to arrive at. Nevertheless, the results allow the conclusion that due to its light weight, the structural dynamic damping efficiency of cork is reasonably high compared to the other approaches presented.

Figure 15. 


\section{Conclusions}

The damping improvement of aluminium honeycomb sandwich structures due to the use of cork inserts was investigated. It is the first time where cork was used as a core insert rather than as a core material. This allows improvement in very stiff, aluminium core sandwich structures. Three different types of structures were analysed: (i) An empty host structure (EHS) without the cork inserts, (ii) a simply damped structure (SDS) for which inserts were distributed equally over the structure, and (iii) structures for which inserts were placed in specific voids to achieve maximum efficiency and hence optimum damping (ODS). The structural dynamic as well as the vibro-acoustic performance was estimated in numerical simulation using ANSYS 16.2 .

In order to assess structural dynamic performance, average squared displacement amplitude reduction efficiency $\overline{E_{\overline{d^{2}}}}$ is defined as target parameter. For vibro-acoustic performance the target parameter is average transmission loss effectiveness $\overline{E_{T L}}$. The structural dynamic models were validated by experimental vibration analysis, whereas the vibro-acoustic models were validated against published data. Different combinations for bonding of the inserts to the host structure were analysed.

Placing only one insert in the centre void is identified as an efficient construction in both structural dynamic and vibro-acoustic performance; an increase in mass of $0.20 \%$ and average squared displacement reduction, $\Delta \overline{d^{2}}$, of $8.46 \%$ could be achieved leading to displacement reduction efficiency, $\overline{E_{\overline{d^{2}}}}$, of 42.44 . On the other hand, the acoustic transmission loss could be increased by $0.6 \mathrm{~dB}$, which results in transmission loss increase efficiency, $\overline{E_{T L}}$, of 51.23. However, highest absolute improvement is obtained with a filling degree of $64 \%$ with an increase in mass of $9.76 \%$; a value of $35.25 \%$ average squared displacement and of $1.5 \mathrm{~dB}$ for transmission loss increase. The TL increase is 2.57 times that achieved by doubling of mass in the mass law region for an acoustic barrier. Interestingly, damping does not increase with the 
number of inserts in a monotonic way due to increased complexity introduced by internal resonances of cork inserts and change in effective mass and stiffness. Additionally, it is shown that improvement does highly depend on spatial distribution of inserts. Comparing the findings against other damping approaches in the literature, cork seems to have slightly inferior energy absorption capability than particles or foam within honeycomb structures. It is important to emphasize, however, unlike cork inserts, previously published results are based on only first few modes. However, due to its very light weight, the damping efficiency is reasonably high for cork inserts. Furthermore, coming from a natural source, cork has the advantage of being a sustainable damping material.

For a true comparison of the current work, vibro-acoustic performance of other published core damping treatments needs to be carried out for higher frequencies. Potentially, to fully assess vibro-acoustic performance of the structures, analysis at higher frequencies, especially, behaviour at the coincidence region as well as the effect of inserts on standing waves within the medium of the voids, which are expected at higher frequencies for honeycombs with larger cell sizes requires further research.

\section{References}

[1] Shin, K. B., Lee, J. Y. and Cho, S. H. (2008) 'An experimental study of low-velocity impact responses of sandwich panels for Korean low floor bus', Composite Structures, 84(3), pp. 228-240.

[2] Banhart, J., Schmoll, C. and Neumann, U. 'Light-weight aluminium foam structures for ships'. Proceedings of the Conference on Materials in Oceanic Environment (Euromat'98), 55-63.

[3] Turner, R. J., Taylor, E. A., McDonnell, J. A. M., Stokes, H., Marriott, P., Wilkinson, J., Catling, D. J., Vignjevic, R., Berthoud, L. and Lambert, M. (2001) 'Cost effective honeycomb and multi-layer insulation debris shields for unmanned spacecraft', International Journal of Impact Engineering, 26(1), pp. 785-796. 
[4] Wang, D. M., Wang, Z. W. and Liao, Q. H. (2009) 'Energy absorption diagrams of paper honeycomb sandwich structures', Packaging Technology and Science, 22(2), pp. 63-67.

[5] Mead, D. J. (1998). Passive Vibration Control. John Wiley \& Sons.

[6] Lakes, R. (1987). Foam Structures with a Negative Poisson's Ratio. Science, 235, pp. 1038-1040.

[7] Panossian, H. (2006). 'Optimized Non-Obstructive Particle Damping (NOPD) Treatment for Composite Honeycomb Structures'. Proceedings of 47th AIAA/ASME/ASCE/AHS/ASC Structures, Structural Dynamics, and Materials Conference.

[8] Woody, S. C. and Smith, S. T. (2006) 'Damping of a thin-walled honeycomb structure using energy absorbing foam', Journal of sound and vibration, 291(1), pp. 491-502.

[9] Scarpa, F. L., Remillat, C., Landi, F. P. and Tomlinson, G. R. 'Damping modelization of auxetic foams'. SPIE's 7th Annual International Symposium on Smart Structures and Materials: International Society for Optics and Photonics, 336-343.

[10] Nia, A. A. and Sadeghi, M. (2010) 'The effects of foam filling on compressive response of hexagonal cell aluminum honeycombs under axial loading-experimental study', Materials \& Design, 31(3), pp. 1216-1230.

[11] Michon, G., Almajid, A., Ferrero, J.-F., Aridon, G., Fascio, V. and Heurteau, J.-P. (2009) 'Dissipation mechanisms identification of soft hollow particle-dampers in honeycomb structures for micro-vibrations environment'.

[12] Wang, B. and Yang, M. (2000) 'Damping of honeycomb sandwich beams', Journal of Materials Processing Technology, 105(1), pp. 67-72.

[13] Wong, C., Daniel, M. and Rongong, J. (2009) 'Energy dissipation prediction of particle dampers', Journal of Sound and Vibration, 319(1), pp. 91-118.

[14] Huang, H., Joe, C.R. and Kim, D.U. (2005). 'Mechanical behavior of rubberfilled multifunctional honeycomb sandwich composite'. Sandwich Structures 7: Advancing with Sandwich, Structures and Materials, pp. 671-680.

[15] Hao, H., Joe, C.-R. and Kim, D.-U. (2005) 'Mechanical behavior of rubberfilled multifunctional honeycomb sandwich composite', Sandwich Structures 7: Advancing with Sandwich Structures and Materials: Springer, pp. 671-680. 
[16] Santos Silva, J., Dias Rodrigues, J. and Moreira, R.A.S. (2010). Application of Cork Compounds in Sandwich Structures for Vibration Damping. Journal of Sandwich Structures and Materials, 12(4), pp.495-515.

[17] Arunkumar, M., Jagadeesh, M., Pitchaimani, J., Gangadharan, K. and Babu, M. L. (2016) 'Sound radiation and transmission loss characteristics of a honeycomb sandwich panel with composite facings: Effect of inherent material damping', Journal of Sound and Vibration, 383, pp. 221-232.

[18] Naify, C. J., Huang, C., Sneddon, M. and Nutt, S. (2011) 'Transmission loss of honeycomb sandwich structures with attached gas layers', Applied Acoustics, 72(2), pp. $71-77$.

[19] Zhou, R. and Crocker, M. J. (2010) 'Sound transmission loss of foam-filled honeycomb sandwich panels using statistical energy analysis and theoretical and measured dynamic properties', Journal of Sound and Vibration, 329(6), pp. 673-686.

[20] Boucher, M.-A., Smith, C., Scarpa, F., Rajasekaran, R. and Evans, K. (2013) 'Effective topologies for vibration damping inserts in honeycomb structures', Composite Structures, 106, pp. 1-14.

[21] Fotsing, E., Sola, M., Ross, A. and Ruiz, E. (2013) 'Lightweight damping of composite sandwich beams: Experimental analysis', Journal of Composite Materials, 47(12), pp. 1501-1511.

[22] Aumjaud, P., Fieldsend, J. E., Boucher, M.-A., Evans, K. E. and Smith, C. W. (2015) 'Multi-objective optimisation of viscoelastic damping inserts in honeycomb sandwich structures', Composite Structures, 132, pp. 451-463.

[23] Castro, O., Silva, J. M., Devezas, T., Silva, A. and Gil, L. (2010) 'Cork agglomerates as an ideal core material in lightweight structures', Materials \& Design, 31(1), pp. 425 432.

[24] Kumar, S. S., Milwich, M., Deopura, B. and Plank, H. (2011) 'Finite element analysis of Carbon composite sandwich material with agglomerated Cork core', Procedia Engineering, 10, pp. 478-483.

[25] Sargianis, J., Kim, H.-i. and Suhr, J. (2012) 'Natural cork agglomerate employed as an environmentally friendly solution for quiet sandwich composites', Scientific reports, 2. 
[26] ANSYS (2015) ANSYS® Academic Research, Release 16.2, Help System, Coupled Field Analysis Guide, ANSYS, Inc.

[27] Colakoglu, M. and Jerina, K. (2003) 'Material damping in 6061-T6511 aluminium to assess fatigue damage', Fatigue \& Fracture of Engineering Materials \& Structures, 26(1), pp. 79-84.

[28] Silva, J., Píriz, M., Gamboa, P., Cláudio, R., Nunes, N. and Lopes, J. 'A passive approach to the development of high performance composite laminates with improved damping properties'. The Eleventh Intern. Conf. on Computational Structures.

[29] Villot, M., Guigou, C. and Gagliardini, L. (2001) 'Predicting the acoustical radiation of finite size multi-layered structures by applying spatial windowing on infinite structures', Journal of sound and vibration, 245(3), pp. 433-455.

[30] Mulholland, K., Parbrook, H. and Cummings, A. (1967) 'The transmission loss of double panels', Journal of Sound and Vibration, 6(3), pp. 324-334.

[31] Bolton, J., Shiau, N.-M. and Kang, Y. (1996) 'Sound transmission through multi-panel structures lined with elastic porous materials', Journal of sound and vibration, 191(3), pp. 317-347.

[32] Brouard, B., Lafarge, D. and Allard, J.-F. (1995) 'A general method of modelling sound propagation in layered media', Journal of Sound and Vibration, 183(1), pp. 129-142.

[33] Zheng, H., Cai, C. and Tan, X. (2004) 'Optimization of partial constrained layer damping treatment for vibrational energy minimization of vibrating beams', Computers \& Structures, 82(29), pp. 2493-2507.

[34] Hou, S. W., Jiao, Y. H., Wang, X., Chen, Z. B. and Fan, Y. B. 'Optimization of plate with partial constrained layer damping treatment for vibration and noise reduction'. Applied Mechanics and Materials: Trans Tech Publ, 20-26.

[35] Mead, D. (1973) 'A general theory of harmonic wave propagation in linear periodic systems with multiple coupling', Journal of Sound and Vibration, 27(2), pp. 235-260.

[36] Mead, D. J. (1970) 'Free wave propagation in periodically supported, infinite beams', Journal of Sound and Vibration, 11(2), pp. 181-197. 
Table 1: Material properties of honeycomb core, aluminium panels and cork inserts.

\begin{tabular}{lcccc}
\hline \multicolumn{1}{c}{ Property } & Unit & Honeycomb core & $\begin{array}{c}\text { Aluminium } \\
\text { panels }\end{array}$ & Cork inserts \\
\hline Series/Grade & & 3003 & $1050 \mathrm{~A}$ & NL 10® \\
Thickness & $\mathrm{mm}$ & 0.05 & 1.2 & 10 \\
Density & $\mathrm{g} / \mathrm{cm}^{3}$ & 2.8 & 2.7 & 0.12 \\
Young's & $\mathrm{GPa}$ & 69 & 71 & 0.0051 \\
Modulus & & & & 0 \\
Poisson's Ratio & & 0.33 & 0.33 & 0.002 \\
Shear Modulus & $\mathrm{GPa}$ & 26 & 26 & \\
\hline
\end{tabular}


Table 2: Initial damping parameters.

\begin{tabular}{ccc}
\hline Damping Parameter & Aluminium $\left(\mathbf{R}^{\mathbf{2}}=\mathbf{0 . 9 9}\right)$ & Cork $\left(\mathbf{R}^{\mathbf{2}}=\mathbf{0 . 9 0}\right)$ \\
\hline$\alpha$ & 3.8078 & 3.4843 \\
$\beta$ & 0 & $5.80 \mathrm{E}-07$ \\
$g$ & 0 & 0.0033 \\
\hline
\end{tabular}


Table 3: Measured damping ratios of the first few modes

\begin{tabular}{ccc}
\hline Mode number & $\begin{array}{c}\text { No additional damping } \\
\text { (EHS) }\end{array}$ & With SDS \\
\hline 1 & 0.0126 & 0.007 \\
2 & 0.0065 & 0.0065 \\
3 & 0.012 & 0.0142 \\
4 & 0.0097 & 0.012 \\
5 & 0.0134 & 0.011 \\
\hline
\end{tabular}


a)

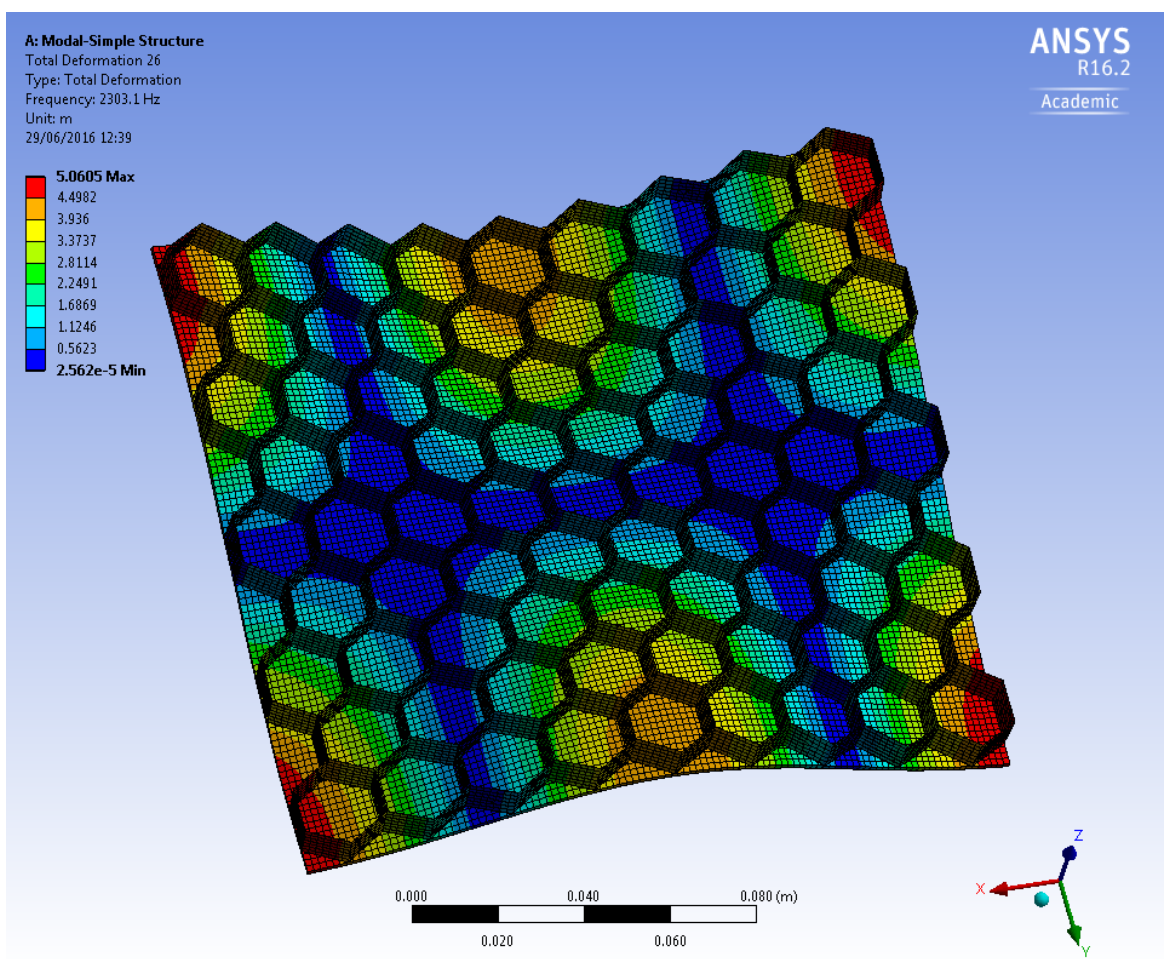

b)

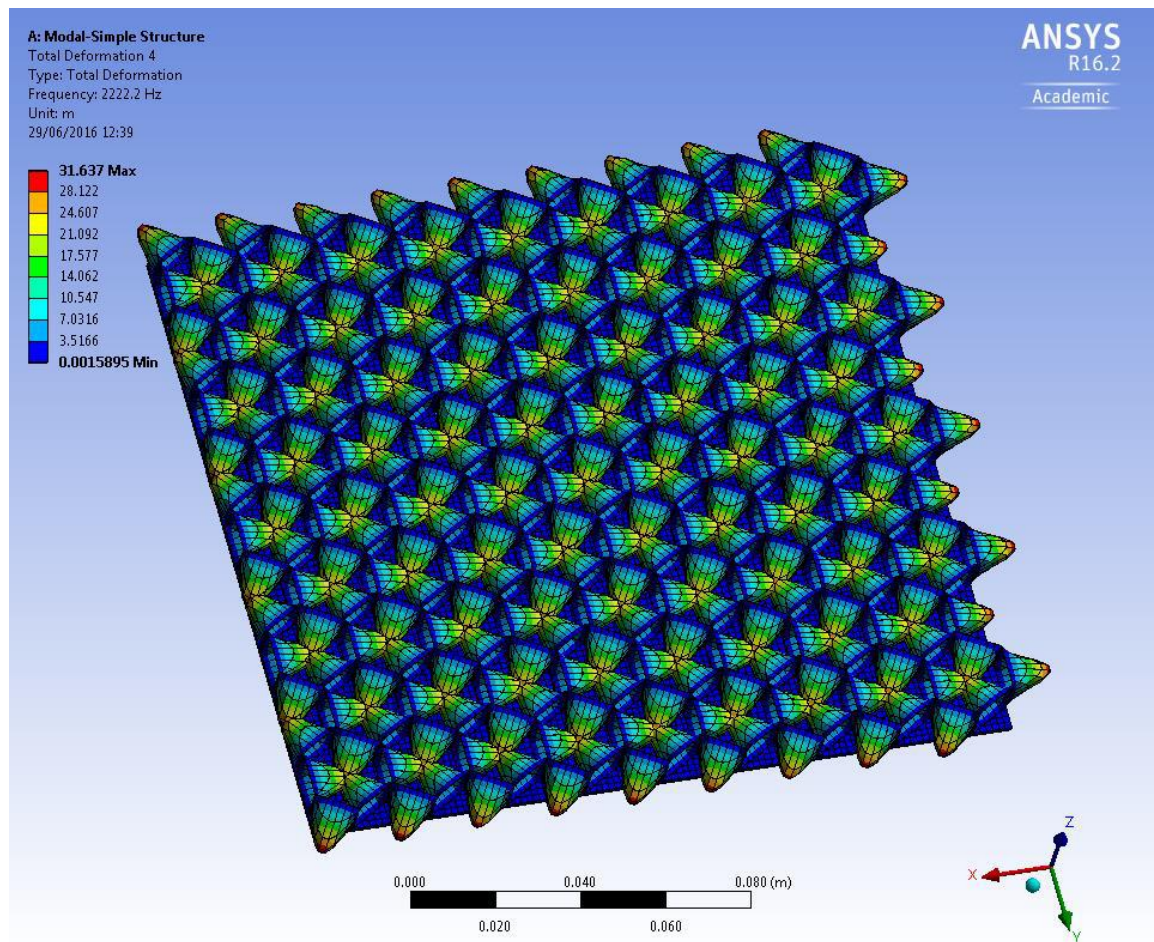

Figure 1. Typical mode shapes of a honeycomb sandwich panels. a) 5th global mode shape which shows bending of the plate and b) 1st local mode shape where core structure shows flexural motion. 
a)

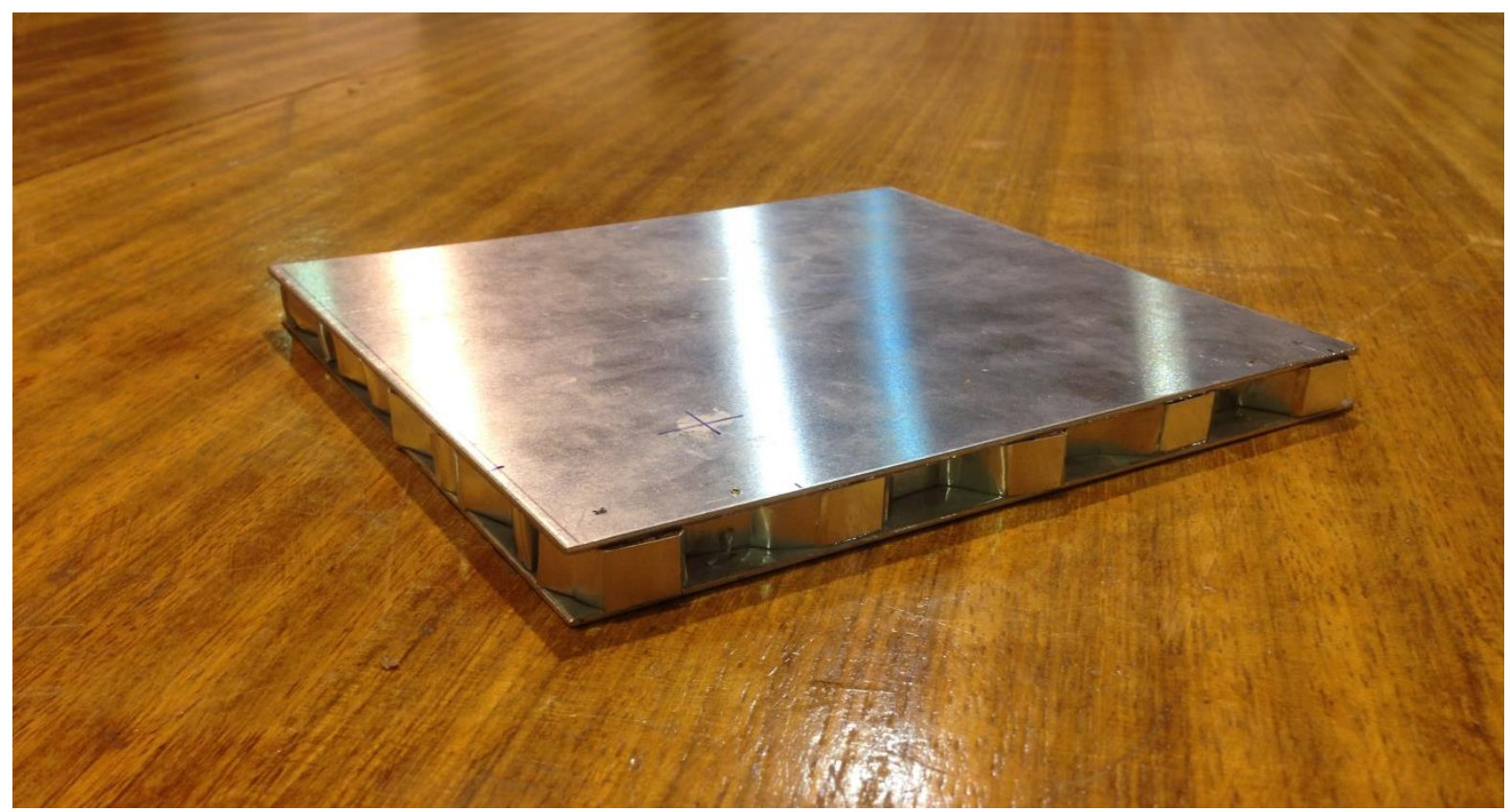

b)

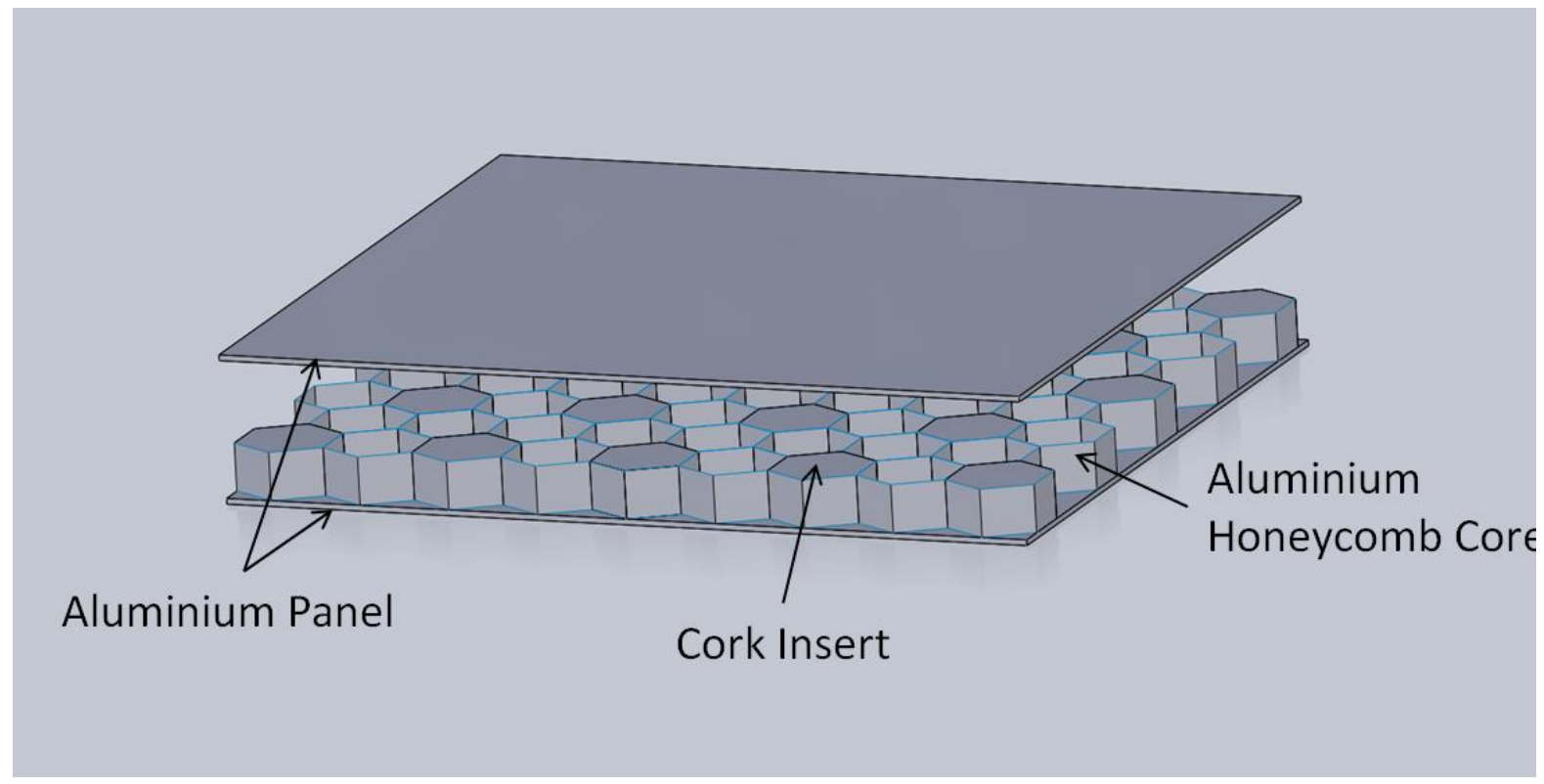

Figure 2: Simply damped structure (SDS) - a) Sample, b) Schematic (Upper panel moved up). 
a)

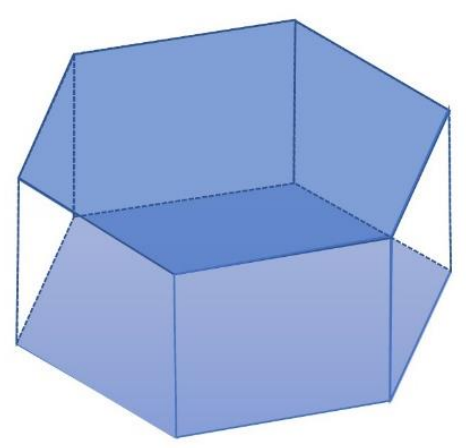

b)

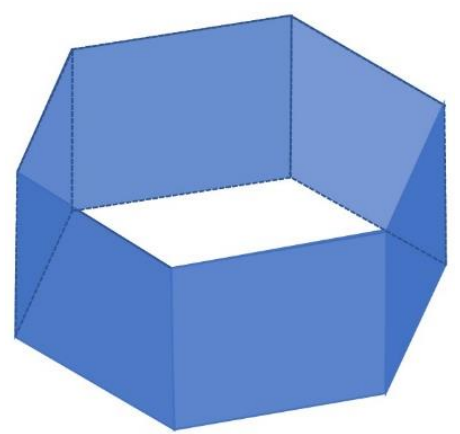

c)

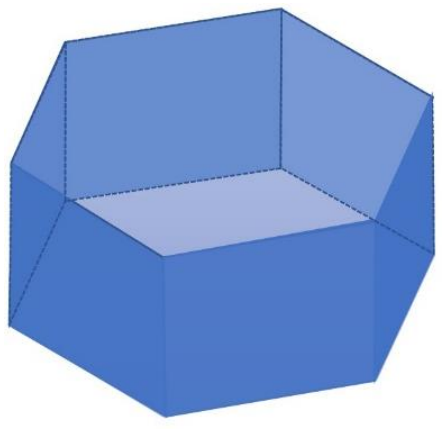

Figure 3: Surface bonding conditions - insert connected to a) Outer panels, b) Core, c) Core and one of the panels. 


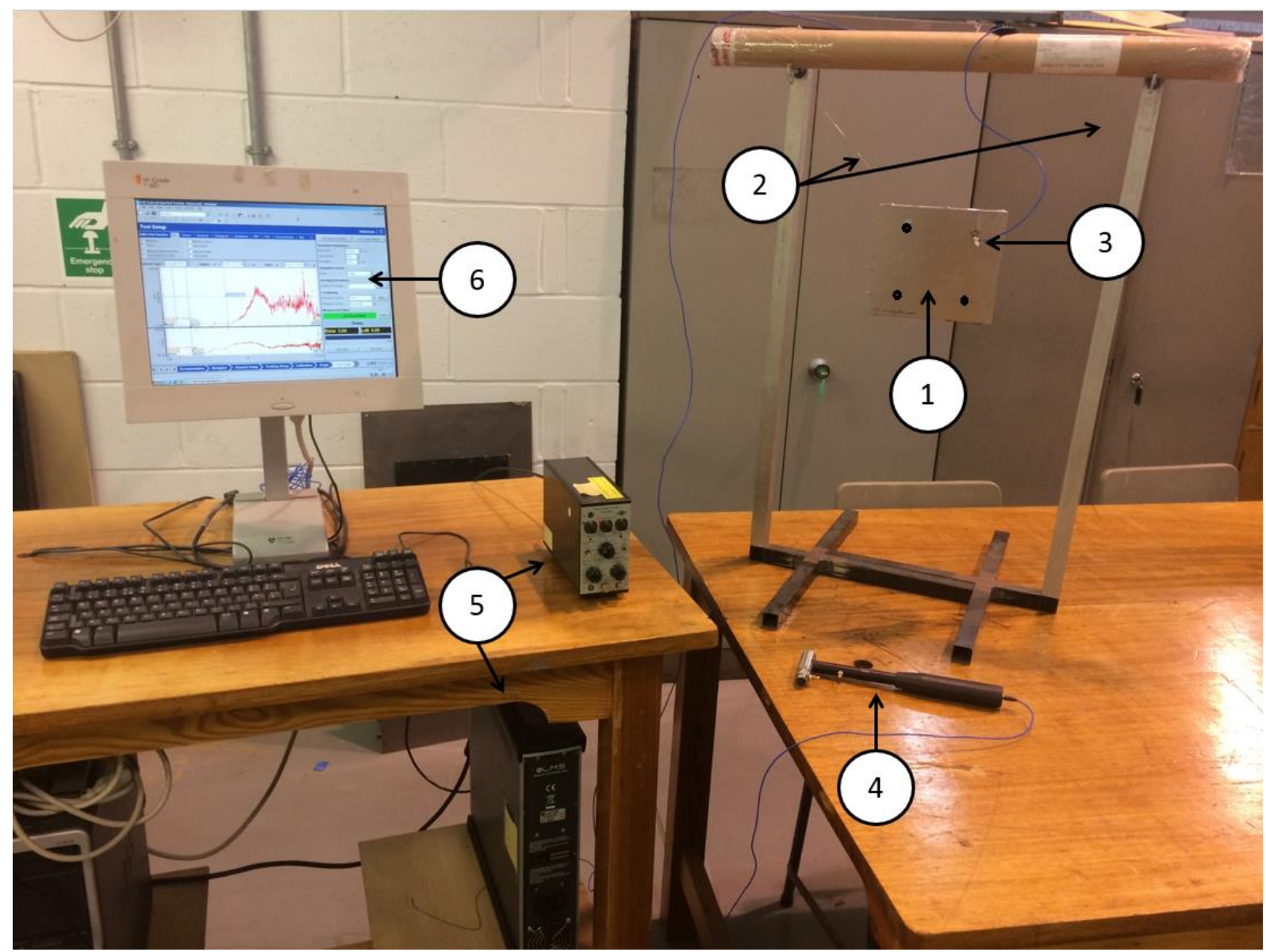

Figure 4: Testing arrangement: 1 - Structure, 2 - Nylon strings, 3 - Accelerometer, 4 - Impact hammer, 5 - Charge amplifier, 6 - PC running analysis software. Th black colour dots on the structure are other response measurement locations. 
a)

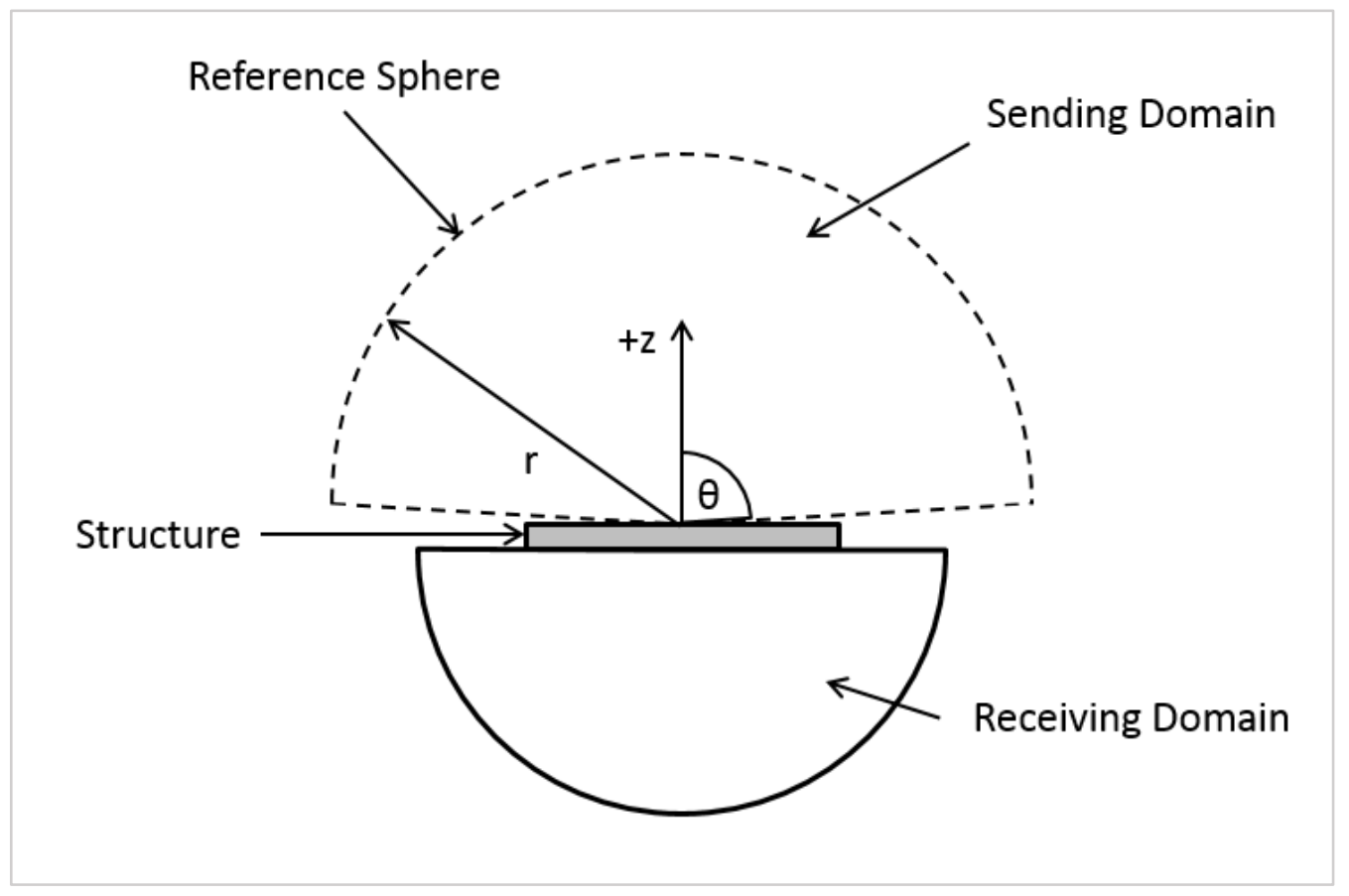

b)

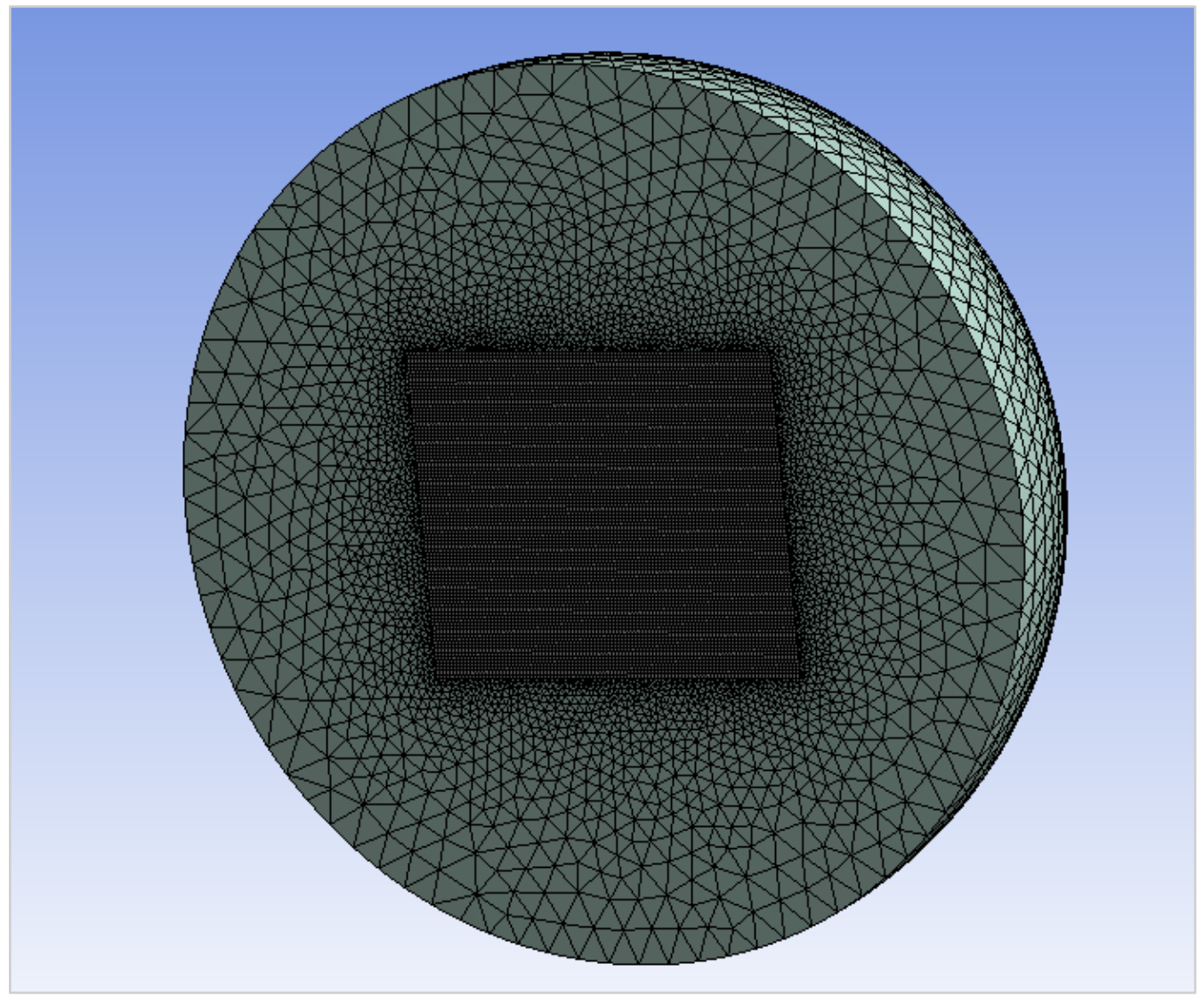

Figure 5: FE model used in numerical vibro-acoustic analysis - a) Schematic and b) Meshed model. 
a)

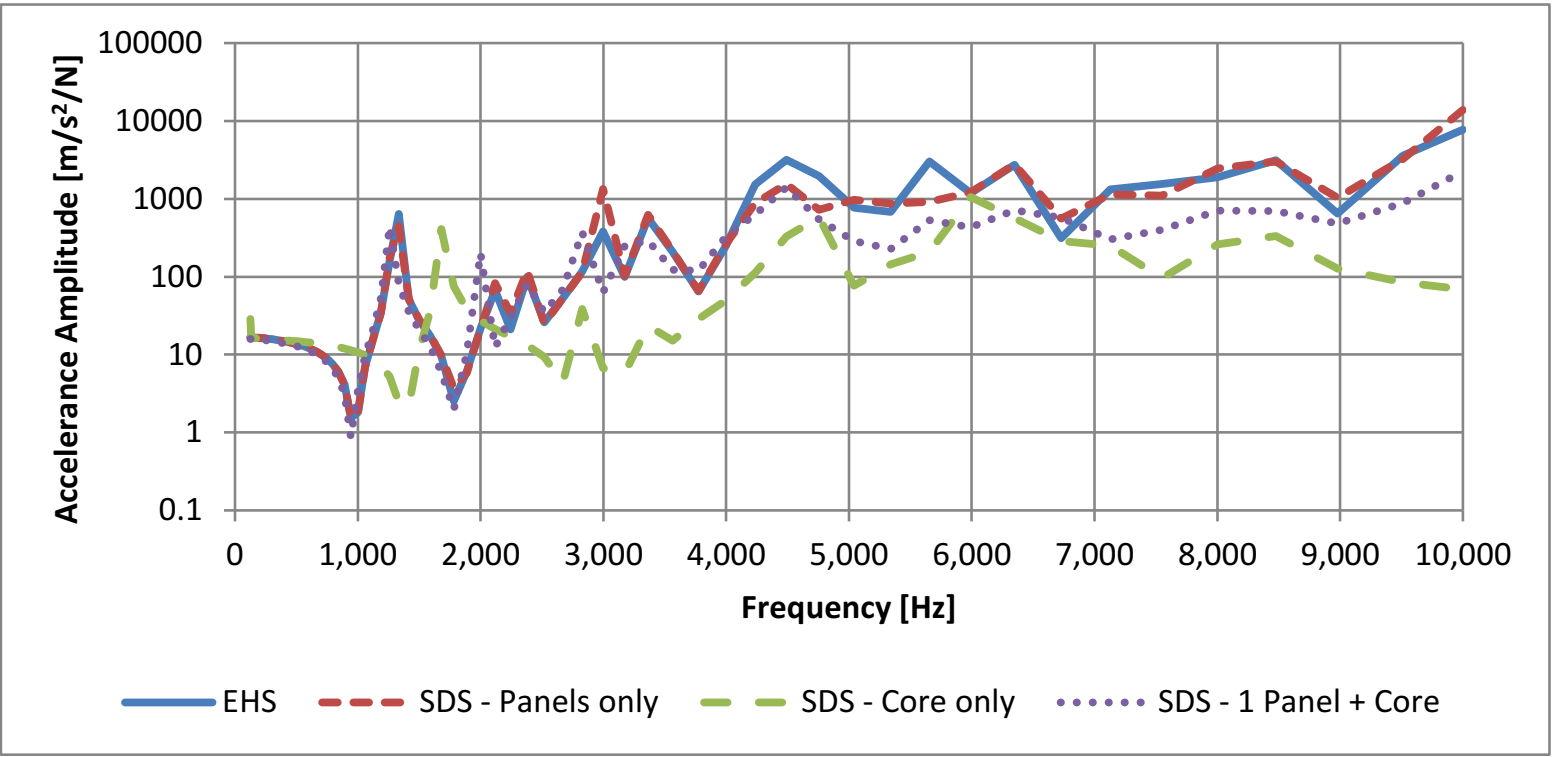

b)

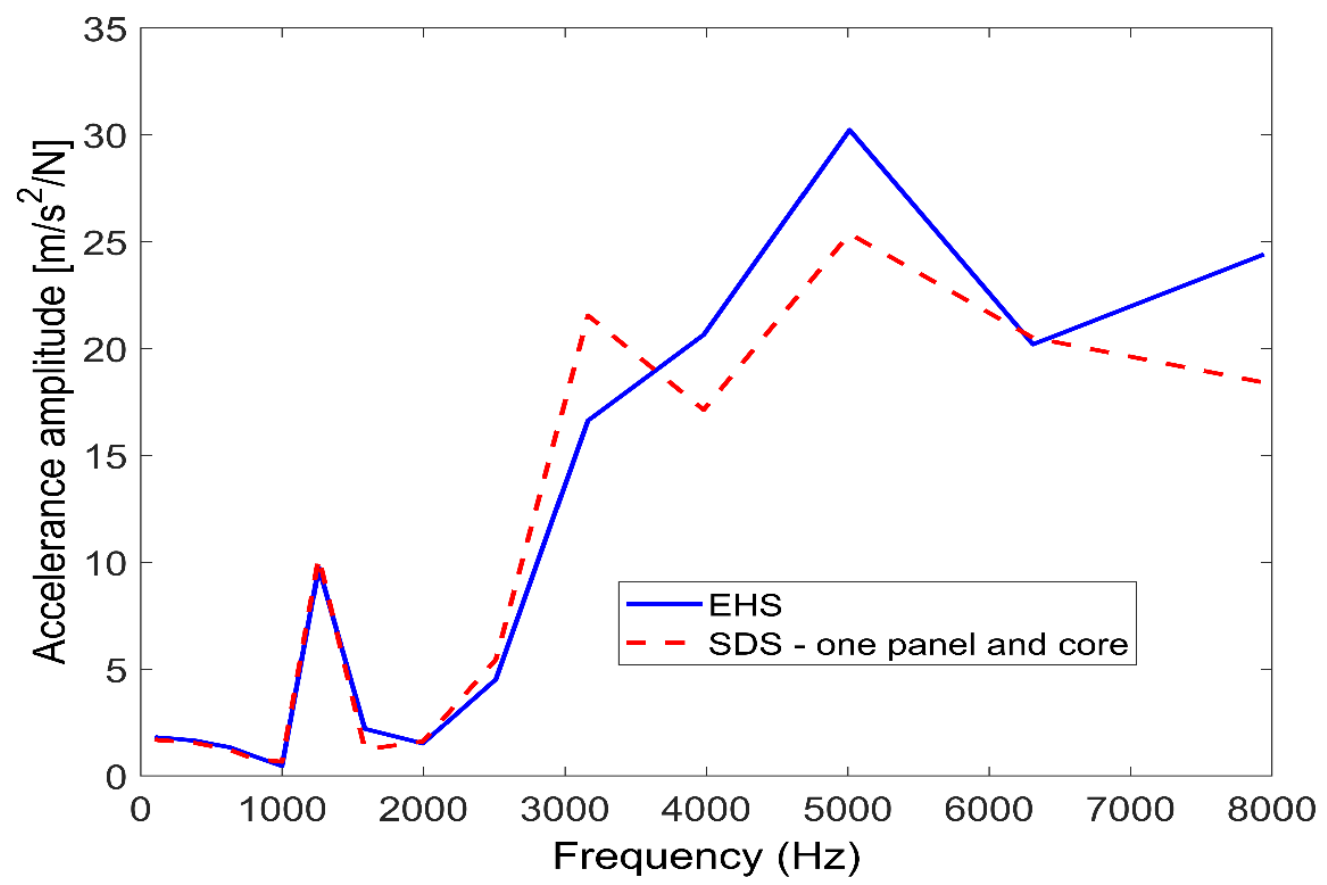

Figure 6: The overall effect of cork inserts. a) numerically simulated accelerance amplitude variation as a function of frequency - different bonding conditions and b) experimental accelerance amplitudes in $1 / 3^{\text {rd }}$ octave bands for with (SDS) and without (EHS) cork inserts. 
a)

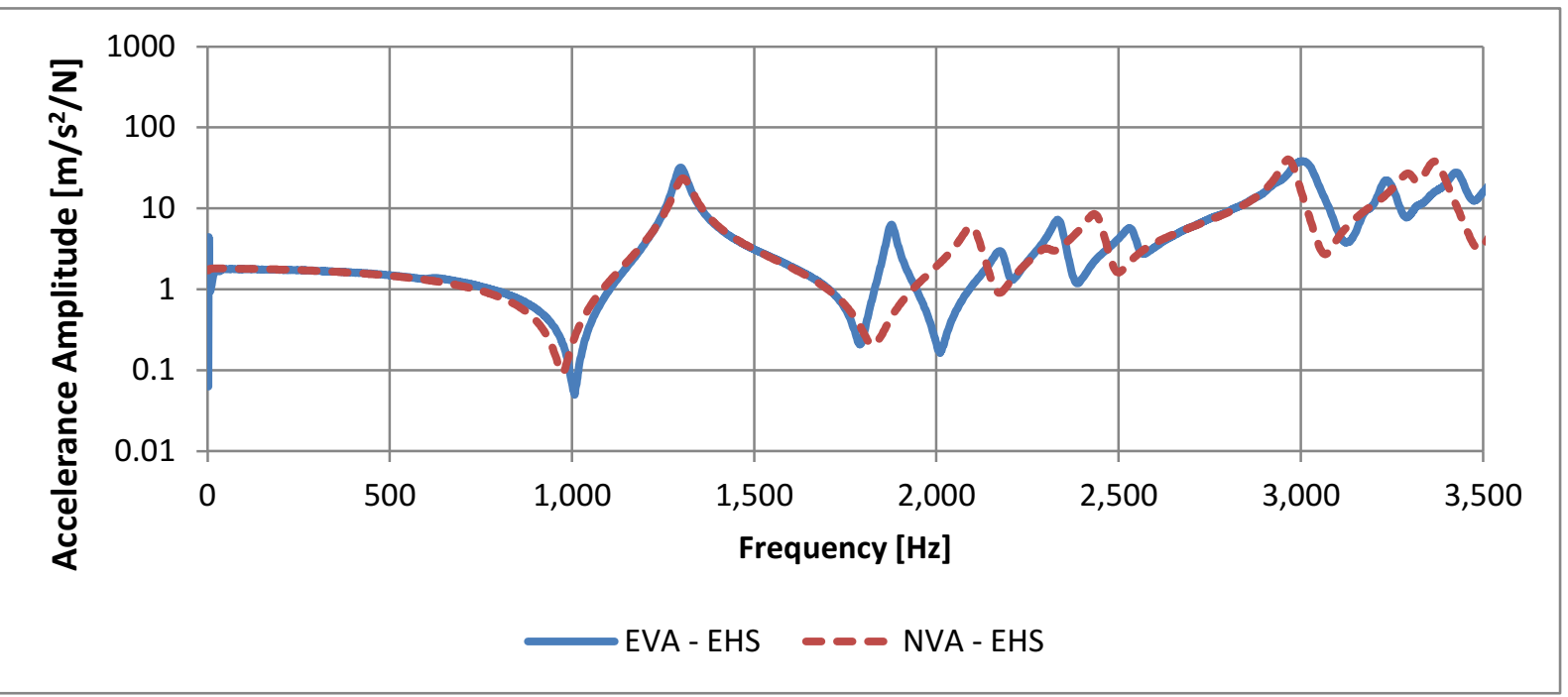

b)

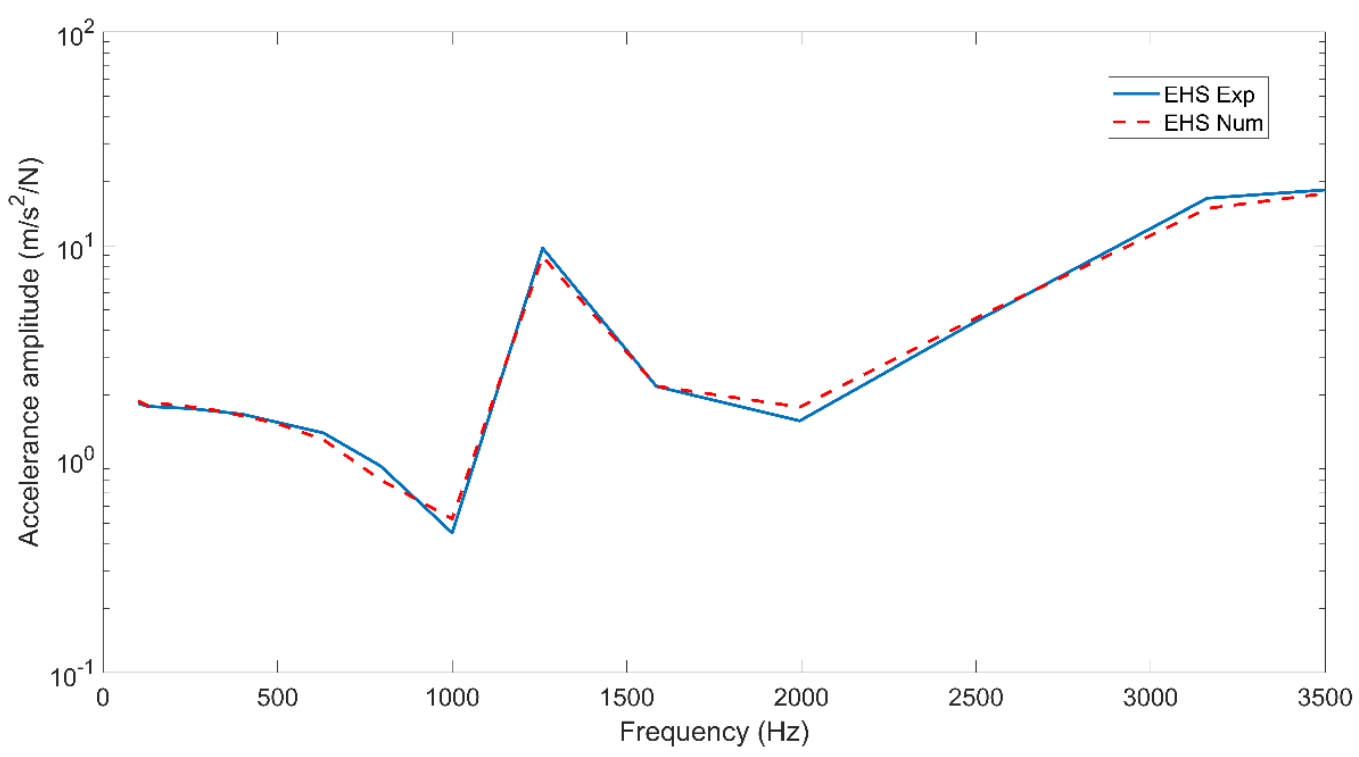

Figure 7: Numerical vibration analysis in comparison with experimental results of structure without any inserts. - a) narrow band representation, experimental and numerical and b) $1 / 3^{\text {rd }}$ octave band representation experimental and numerical . 
a)

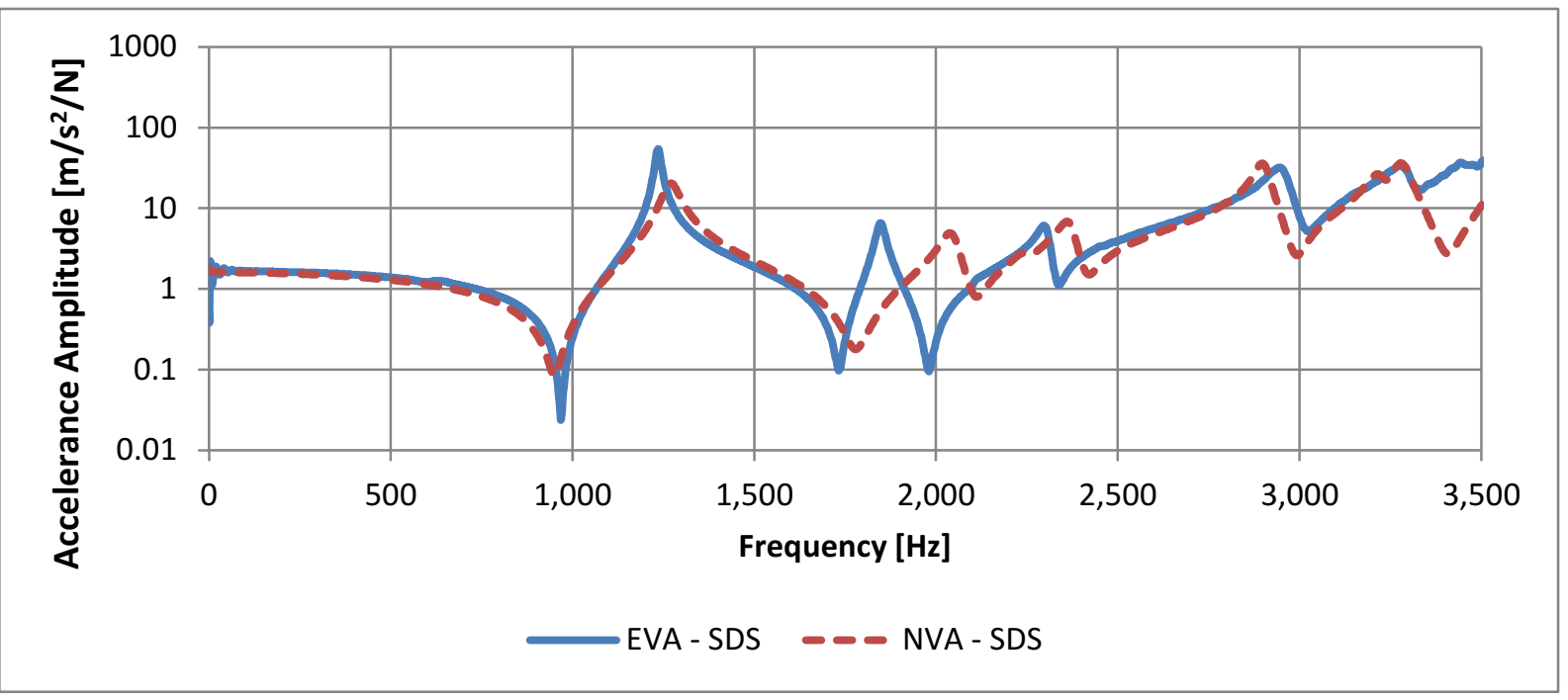

b)

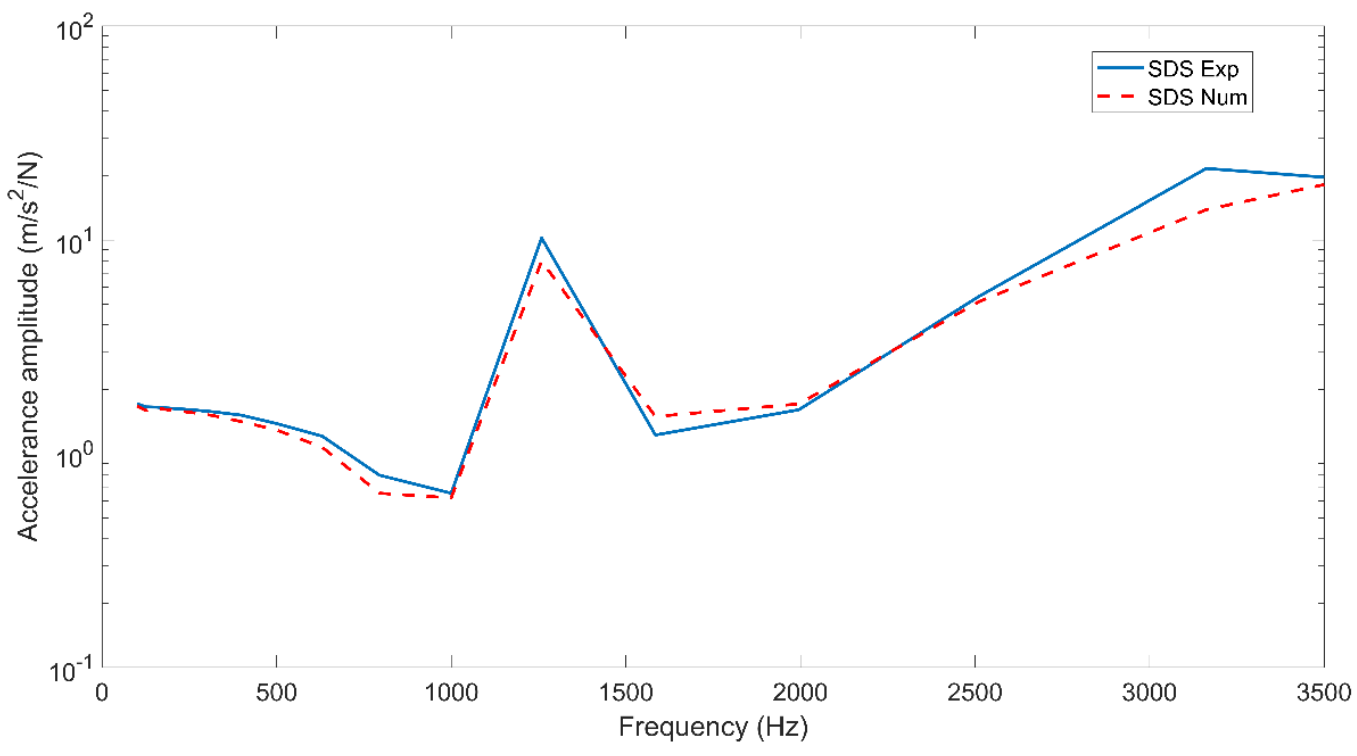

Figure 8: Numerical vibration analysis in comparison with experimental results for structure with inserts a) narrow band representation, experimental and numerical and b) $1 / 3^{\text {rd }}$ octave band representation, experimental and numerical. 


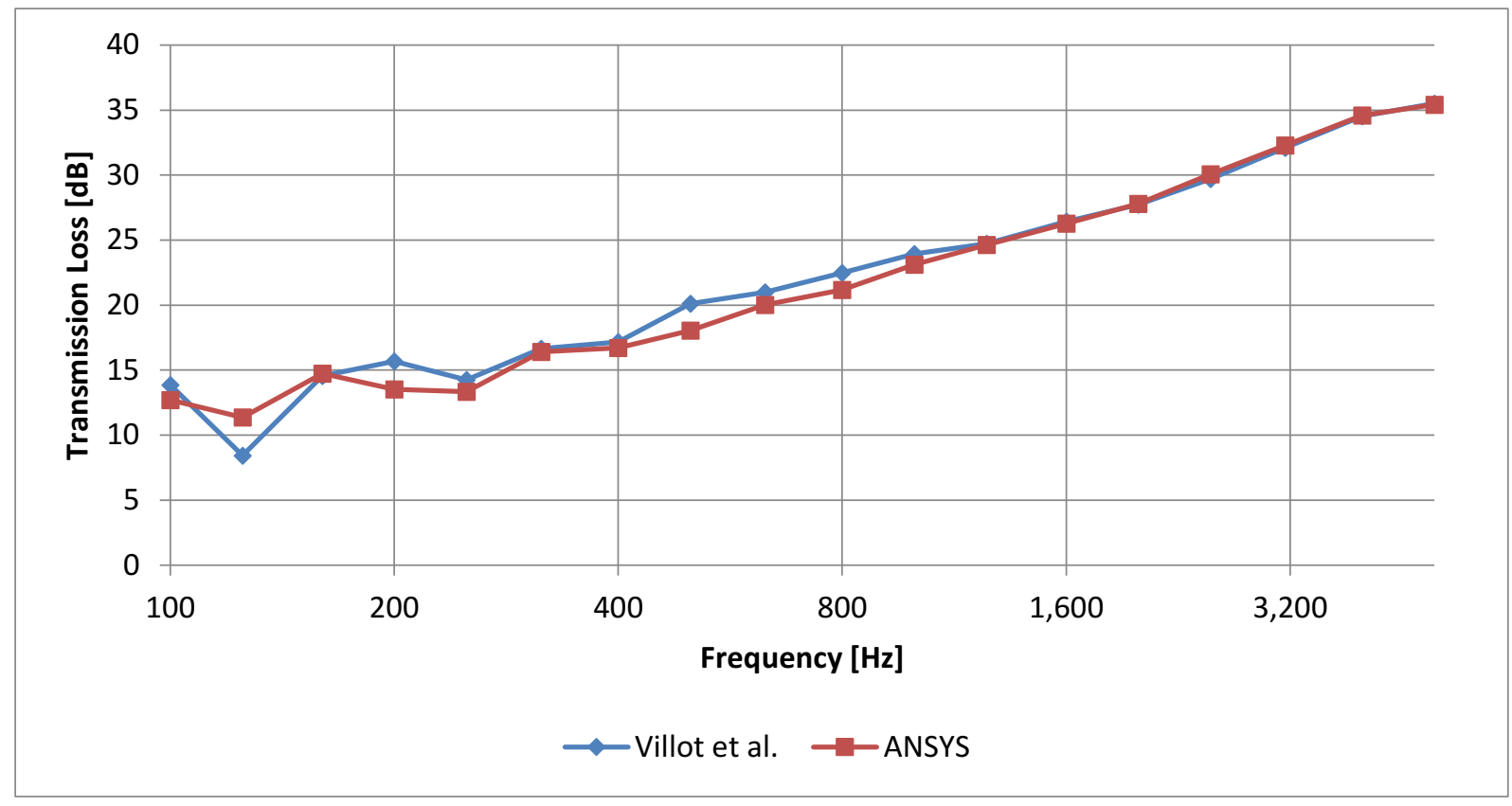

Figure 9: Validation of vibro-acoustic model - comparison of estimated transmission loss with published data. 
a)

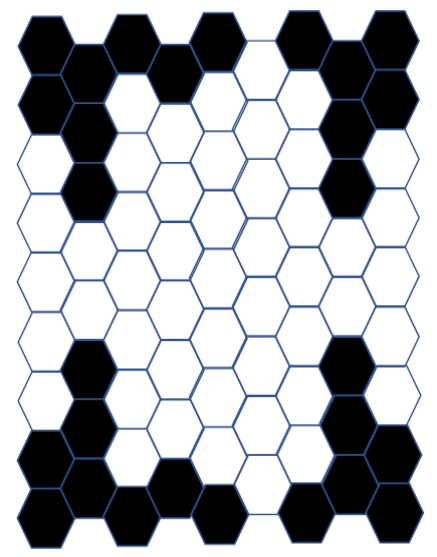

b)

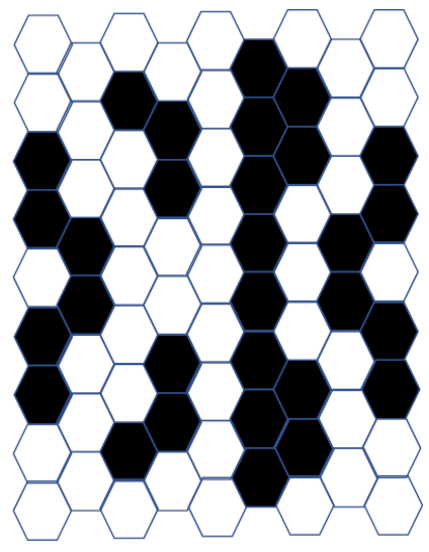

d)

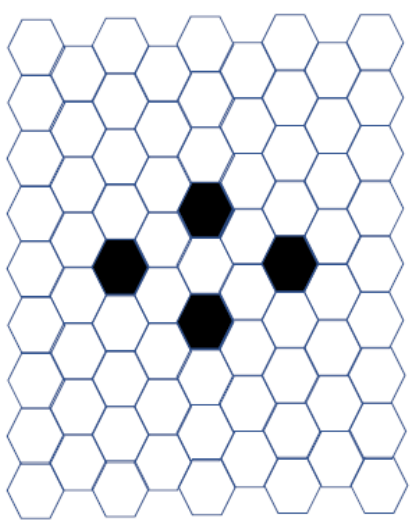

c)

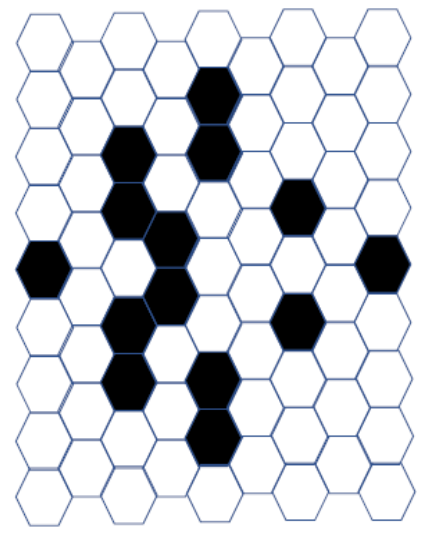

e)

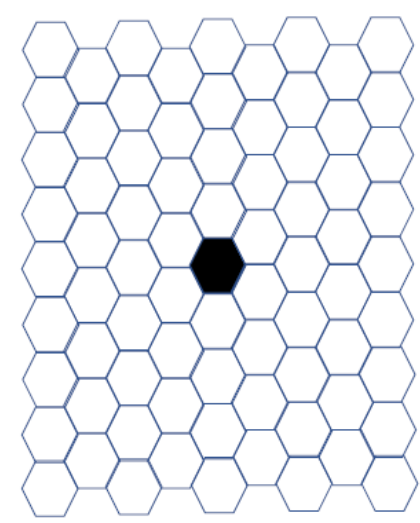

Figure 10: Arrangement of inserts in clusters for optimisation study. a) Cluster 1 - CL1; b) Cluster 2 CL2, c) Cluster 3 - CL3; d) Cluster 4 - CL4 and e) Cluster 5 - CL5. 


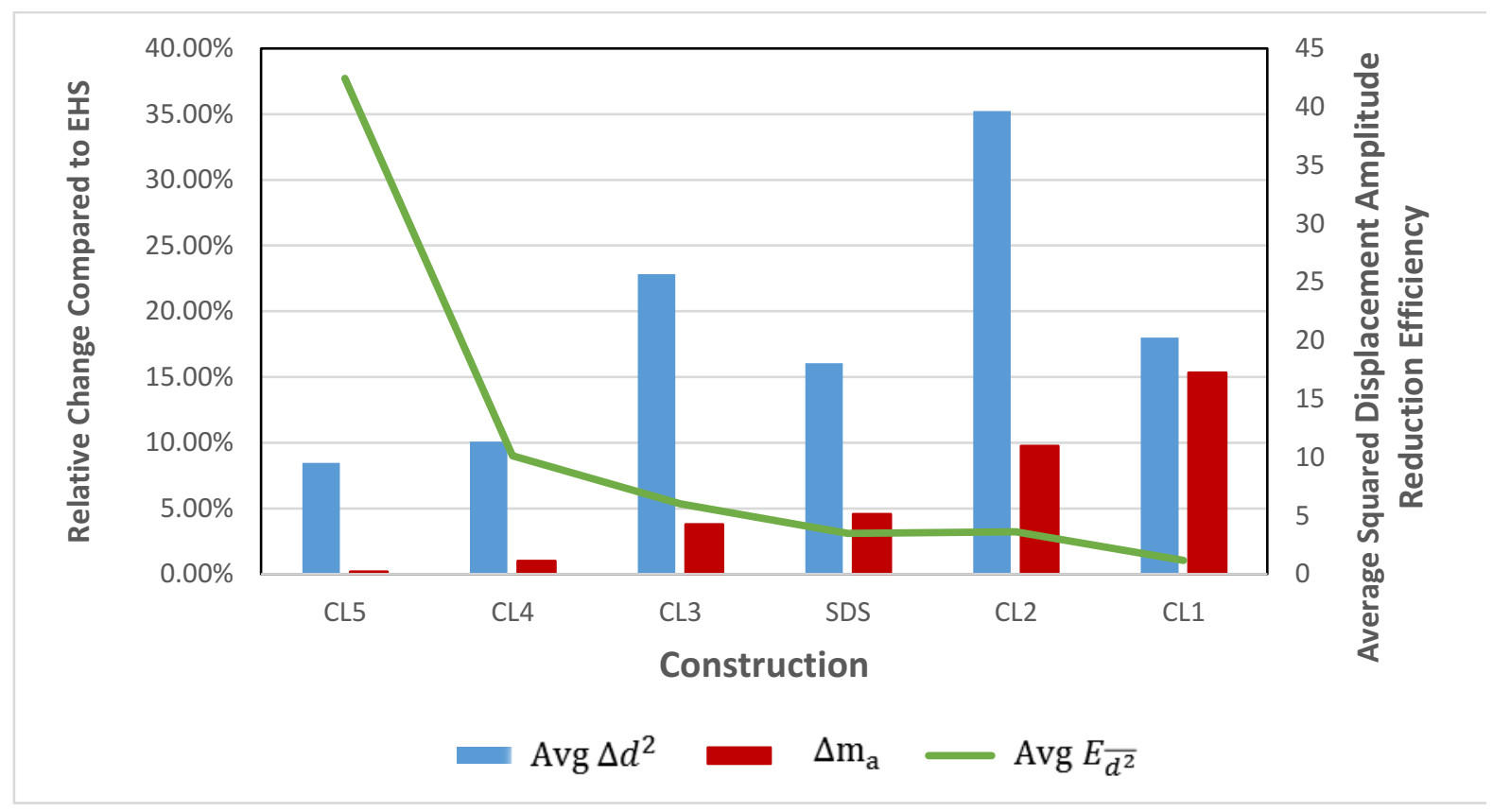

Figure 11: Comparison of structural dynamic performance of different clusters in relation to the EHS in order of filling degree. Avg $\Delta d^{2}$ - average squared displacement reduction, $\Delta m_{\mathrm{a}}$ - increase in mass and Avg $E_{\bar{d}^{2}}$ - Average displacement decrease efficiency. 
a)

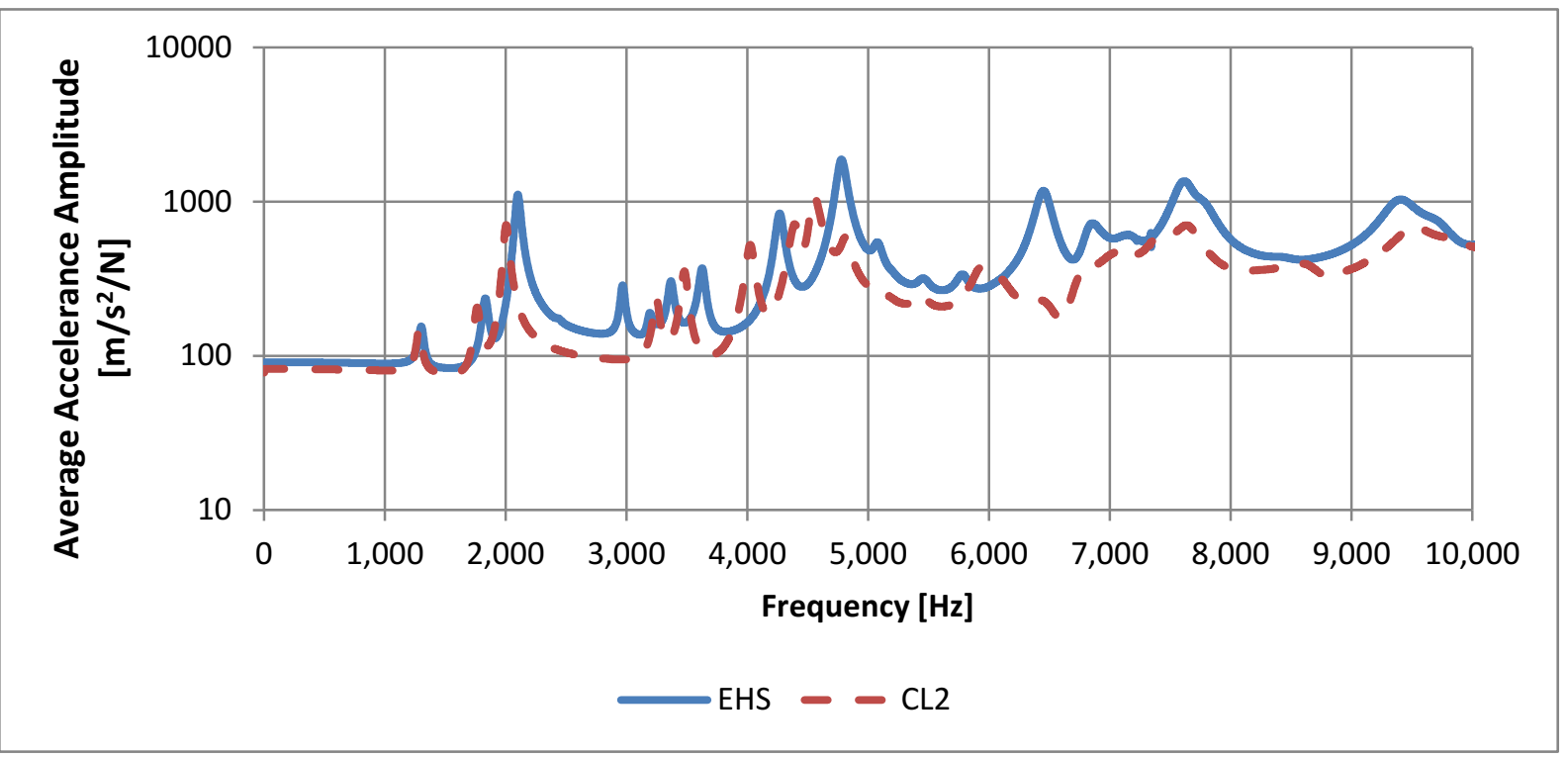

b)

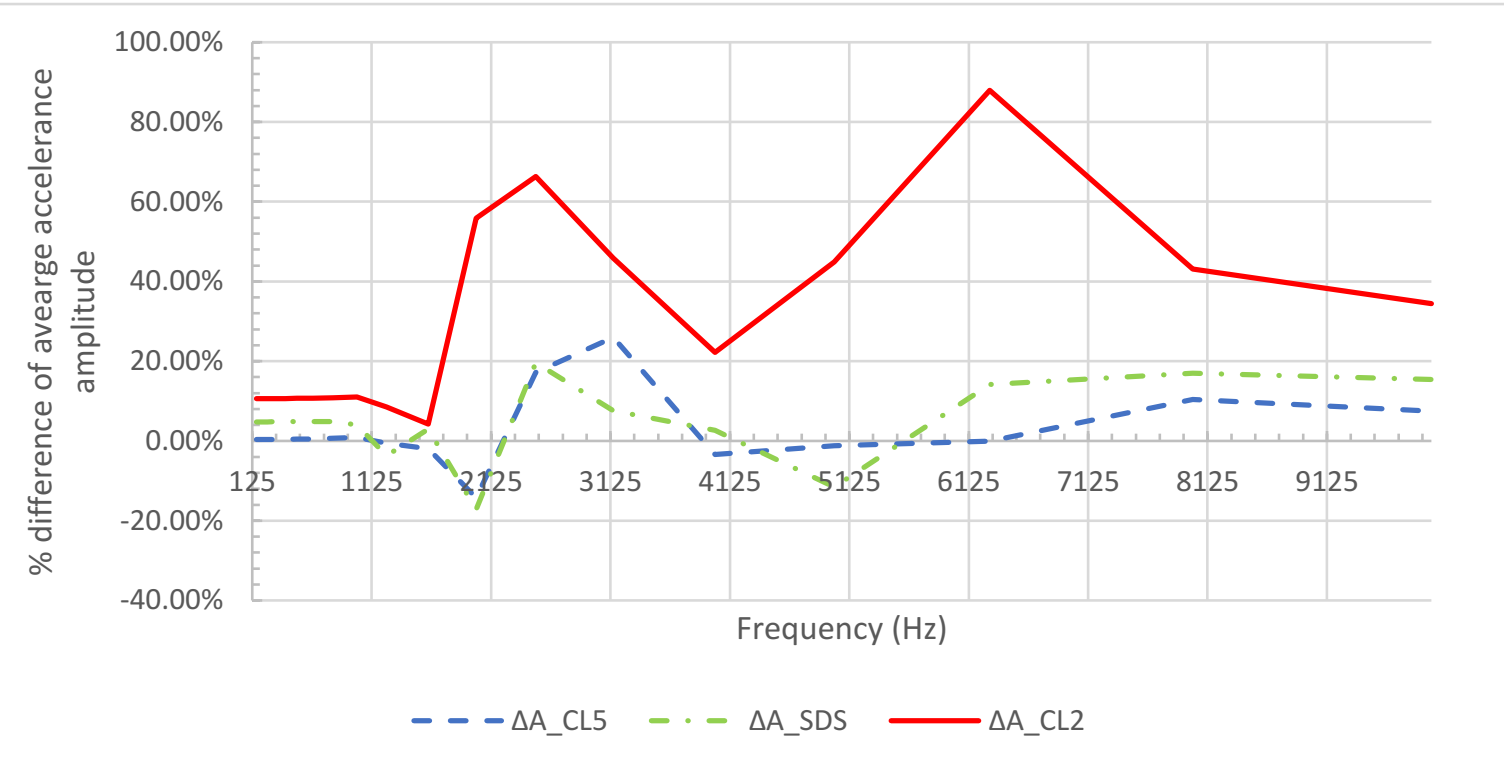

Figure 12: Structural dynamic performance of different clusters. a) space averaged accelerance amplitude of EHS and CL2, b) relative change in acceleration response compared to the EHS - 1/3 octave bands. 

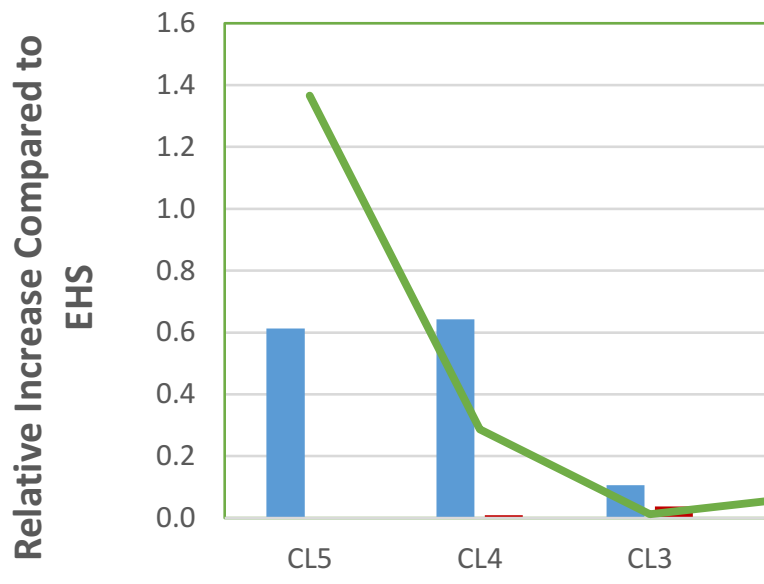

CL3

Construction

Figure 13: Comparison of vibro-acoustic performance in relation to the EHS - in order of filling degree. Avg $\Delta \mathrm{TL}$ - average increase in transmission loss in $\mathrm{dB}, \Delta m_{\mathrm{a}}$ - increase in mass and $\mathrm{Avg} E_{\Delta \mathrm{TL}}$ average transmission loss increase efficiency. 
a)

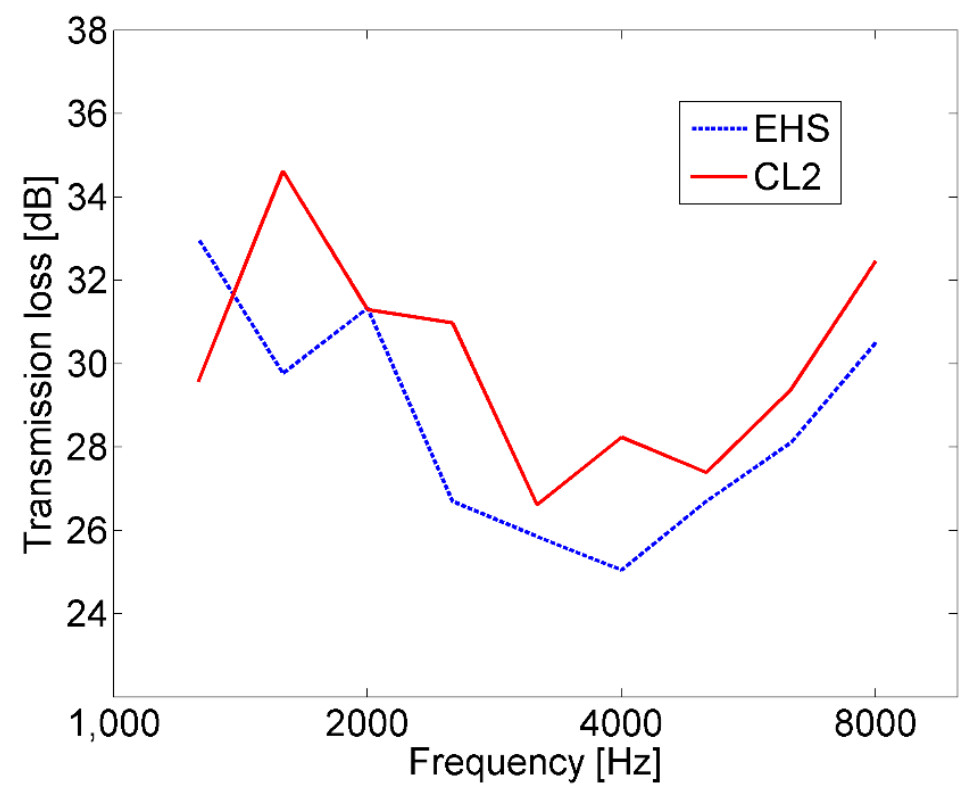

b)

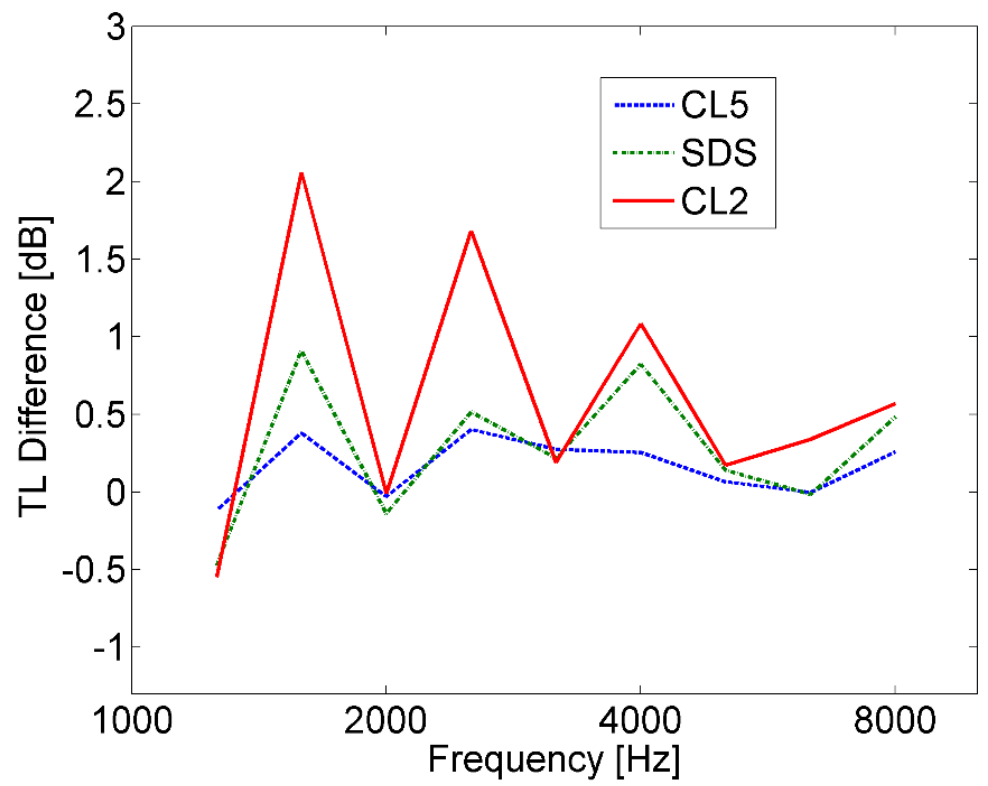

Figure 14: Vibro-acoustic performance of different clusters. a) average transmission loss of EHS and CL2, and b) relative change in transmission loss compared to the EHS in 1/3 octave bands. 
a)

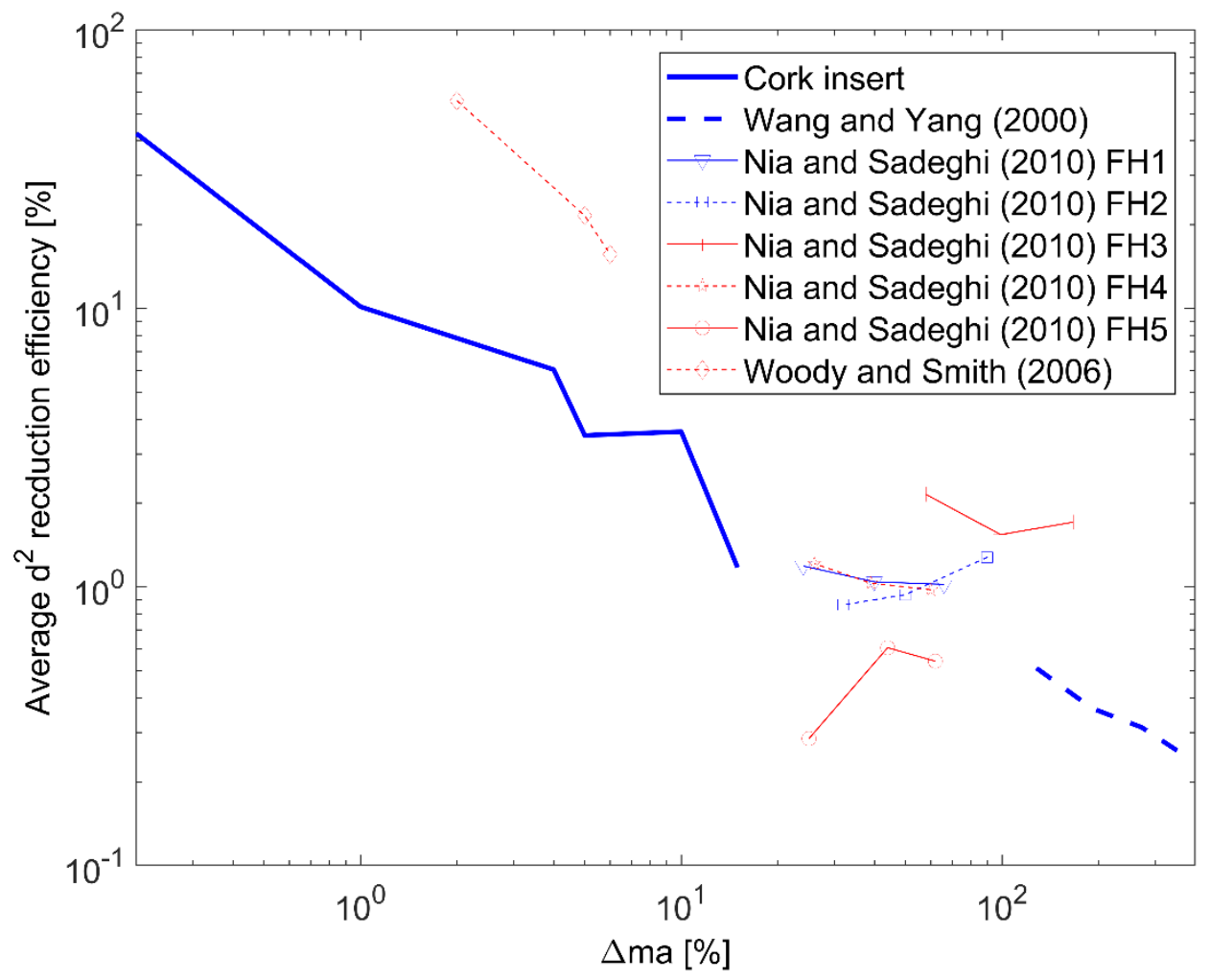

b)

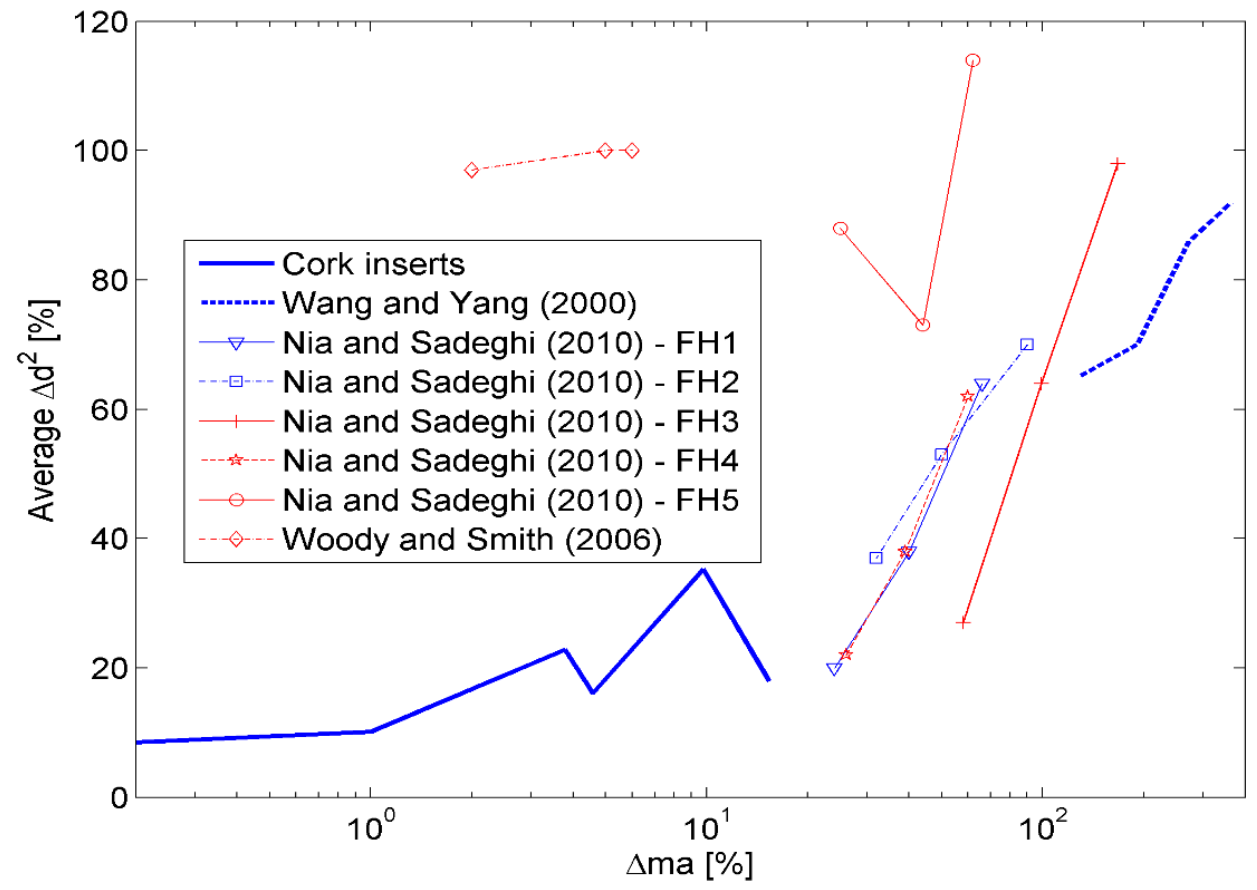

Figure 15: Comparison of structural dynamic performance for different damping approaches. - a) $\overline{E_{\overline{d^{2}}}}$ over $\Delta m_{a}$, b) $\overline{\Delta \overline{d^{2}}}$ over $\Delta m_{a}$. 